\title{
A PARTIAL DESCRIPTION OF THE PARAMETER SPACE OF RATIONAL MAPS OF DEGREE TWO: PART 2
}

\author{
Mary Rees \\ Department of Pure Mathematics, University of Liverpool, \\ P.O. Box 147, Liverpool L69 3BX, U. K. \\ Address until 30th June 1991: \\ Institute of Mathematical Sciences, SUNY at Stony Brook, \\ Stony Brook NY 11794-3660, U.S.A.
}

\begin{abstract}
This continues the investigation of a combinatorial model for the variation of dynamics in the family of rational maps of degree two, by concentrating on those varieties in which one critical point is periodic. We prove some general results about nonrational critically finite degree two branched coverings, and finally identify the boundary of the rational maps in the combinatorial model, thus completing the proofs of results announced in Part 1.
\end{abstract}




\section{Introduction.}

This is the second in a series of papers aimed at understanding the variation in dynamical behaviour within the family of rational maps of degree two. This introduction will be brief, as Chapter 1 of [R2] was intended as an introduction to this paper also, and includes statements of the results of this paper.

As in [R2], we concentrate on maps which are critically finite. Recall that an orientation-preserving branched covering $g: \overline{\mathbf{C}} \rightarrow \overline{\mathbf{C}}$ is called critically finite if $\#(X(g))<\infty$, where

$$
X(g)=\left\{g^{n}(c): c \text { critical, } n>0\right\},
$$

and that these play an important rôle in the understanding of rational maps. A branched covering of $\overline{\mathbf{C}}$ of degree two necessarily has two critical points $c_{1}$, $c_{2}$. More generally, we consider degree two branched coverings for which one critical point is periodic. Likewise, these play an important rôle. Douady and Hubbard have studied the Mandelbrot set for degree two polynomials (for which the critical point at $\infty$ is, of course, fixed). They provided a combinatorial model for the Mandelbrot set ([D-H1], [D-H2]) which was reinterpreted by Thurston $[\mathbf{T}]$. The work described in this paper, and its predecessor and successors, is a generalisation of the combinatorial model of the Mandelbrot set to maps with a critical point which is no longer fixed, but still periodic. It seems likely that this model can be completed to include maps for which both critical points have infinite forward orbits.

Recall that two critically finite branched coverings $f, g$ are equivalent, written $f \simeq g$, if there exists an orientation-preserving homeomorphism $\varphi: \overline{\mathbf{C}} \rightarrow \overline{\mathbf{C}}$ and a path $g_{t}(t \in[0,1])$ through critically finite branched coverings such that $X\left(g_{t}\right)=X(g)$ for all $t \in[0,1], \varphi \circ f \circ \varphi^{-1}=g_{0}, g=g_{1}$. Thurston [T] gave a necessary and sufficient condition for a critically finite branched covering to be equivalent to a rational map. Almost always - and always in degree two - there is at most one rational map in an equivalence class, and the rational map can be determined up to topological conjugacy (and its Julia set, up to homeomorphism) by any map in the equivalence class. More detail is given on this in [R2].

We are interested in understanding rational maps, but, paradoxically, the subject of this paper is, very largely, critically finite branched coverings which are not equivalent to rational maps. The reason is basically the following. Roughly speaking, it is easier to describe dynamically the family of all critically finite degree two branched coverings with, say, one critical point of period $m$ than simply the family of all such rational maps. (We remark that there is not so much difference between the two families if $m=1$.) It is also easier to analyse nonrational branched coverings (up to equivalence) rather than rational maps, and then, of course, the rational ones are what remain when the nonrational ones are removed.

The chapters of this paper give increasingly precise results about increasingly specified branched coverings. The last two chapters concern lamination 
maps, which are, for the present, our preferred models for rational maps. Their dynamics are easily computable, and a lamination map determines an equivalent rational map up to topological conjugacy. This is the substance of the Lamination Map Conjugacy Theorem in [R2]. The chapters, and their rough content, are as follows.

Chapter 1: Homotopy Information about Branched Coverings of Degree Two.

Thurston's theorem giving a necessary and sufficient condition for equivalence of a critically finite branched covering $f$ to a rational map ([T], [D-H3]) is in terms of the action of $f^{-1}$ on isotopy classes of loops in $\overline{\mathbf{C}} \backslash X(f)$. This condition, and a couple of refinements in degree two, which are essentially isotopyinvariance conditions, are stated in the introduction of [R2]. This chapter examines more closely the structure of $f$ when $f$ is not equivalent to a rational map. In particular, a critical branched covering is associated to $f(1.9)$, essentially a restriction of an iterate of $f$ to a subsurface. It may be degree one or two, and is in some sense irreducible. The main result of the chapter is the Decomposition Proposition of 1.12 .

Chapter 2: The Fixed Holed-Sphere Theorem.

The isotopy-invariance of the first chapter is translated into actual invariance, in the Fixed Holed-Sphere Theorem. Underlying this is an application of the common idea that a map with homotopy type satisfying certain "expanding" or "hyperbolic" properties is determined up to a useful semiconjugacy by its homotopy type.

Chapter 3:1-dimensional Topological Dynamics.

No particular originality is claimed for this. The 1-sided Neighbourhood Theorem is similar (though not identical, so far as I know) to many in the literature, and shows, roughly, that under certain mild conditions, a graph preserved by a map is a union of unstable manifolds.

Chapter 4: The Nonrational Lamination Map Theorem.

Now we specify to lamination maps, and show that an invariant lamination with a critically finite lamination map with a nondegenerate Levy cycle (roughly speaking, not equivalent to a rational map) contains periodic leaves - with a specified bound on the period - with certain additional properties. Finally, this result is used in

Chapter 5: Proof of the Admissible Boundary Theorem.

Lamination maps (at least, those which have been defined at any point in this work) have 0 as a periodic critical point, and lie in countably many families, depending on the definition of the map on a set containing the orbit of 0 . The lamination maps in each family are identified with leaves or complementary components - called gaps - of a lamination on a disc, called a parameter lamination and denoted by $\mathcal{L}_{r}$, where $r$ runs through the odd denominator rationals in $(0,1)$. For details of this structure, see Chapter 1 of [R2]. This chapter contains the proof of two results stated in the introduction of [R2] - the Admissibil- 
ity Proposition and the Admissible Boundary Theorem. Identifying lamination maps with the corresponding leaves and gaps in the laminated disc, these two theorems together identify an open set of lamination maps (and its boundary). In this set, all critically finite branched coverings have at most only degenerate Levy cycles, which virtually means they are all equivalent to rational maps, and the set contains all critically finite rational maps, up to equivalence. 
Chapter 1. Homotopy information about Branched Coverings of Degree Two.

1.1 .

The information in this chapter is not very deep, and most of it is known anyway: it is repeated for convenience. Let $f: \overline{\mathbf{C}} \rightarrow \overline{\mathbf{C}}$ be a critically finite degree two orientation-preserving branched covering. Then exactly one of the following occurs, as can be extracted from [T] , and is explained in $1.2-1.3$ below.

Up to equivalence, $f$ is a rational map.

Up to equivalence, $f$ is $[x] \mapsto[A x+b]: T^{2} / \sim \rightarrow T^{2} / \sim$, where $T^{2}$ is the two-torus, $\sim$ is the relation $x \sim-x,[x]$ is the equivalence class of $x, b \sim-b$ and $A$ is an automorphism of $T^{2}$ with determinant 2 and eigenvalues $\lambda_{s}, \lambda_{u}$, $\left|\lambda_{s}\right|<1<2<\left|\lambda_{u}\right|$.

There is a Levy cycle for $f$. (This term is recalled below.)

The third possibility is in some sense a decomposable case. We shall define a complete Levy set for $f$, consisting of a union of disjoint Levy cycles and some preimages of these. Given $f$ and a complete Levy set $\Gamma$, we shall see that components of $\overline{\mathbf{C}} \backslash \cup \Gamma$ are eventually periodic in a natural sense, and hence have maps associated to them, which are always either homeomorphisms or degree two critically finite branched coverings. In particular, such a map is associated to the component of $\overline{\mathbf{C}} \backslash \cup \Gamma$ containing both critical points, in the case when the forward orbits of both critical points of $f$ contain periodic critical points, and this map is of one of the first two indecomposable types if it is degree two. We shall summarise most of the information in the Decomposition Proposition (1.12). This information will be crucial in the semiconjugacy results for critically finite degree two branched coverings, in Chapter 2. It is clear that a more complete description of those critically finite degree two branched coverings with Levy cycles is possible, partly in terms of a natural tree obtained by treating Levy loops as vertices, and partly in terms of the maps associated with components of $\overline{\mathbf{C}} \backslash \cup \Gamma$. I suppose the description would be reminiscent of the Douady-Hubbard-Thurston description of critically finite quadratic polynomials, with some additional identifications. In fact, Shishikura $[\mathbf{S}]$ has some results in a more general framework: he has constructed a tree (with an expanding metric) using Thurston obstruction loops as vertices.

\subsection{The cases when a Thurston obstruction does not exist.}

The Thurston obstruction is a necessary and sufficient condition for nonequivalence to a rational map, when a certain orbifold $Q_{g}$, associated to an orientationpreserving critically finite branched covering $g$, is hyperbolic ([T] Ch. 16). Without going into too much detail about what this means, we identify those $g$ of 
degree two which are not equivalent to rational maps, and for which $Q_{g}$ is not hyperbolic. If $Q_{g}$ is not hyperbolic, then $\#(X(g)) \leq 4$ ([T] Ch. 16).

If $g$ and $h$ are critically finite orientation-preserving branched coverings and $\#(X(g)) \leq 3$, then $g$ and $h$ are equivalent if and only if there is an isomorphism between $\left(g^{-1} X(g), g\right)$ and $\left(h^{-1} X(h), h\right)$ which maps critical points to critical points of the same multiplicity. This follows from the fact that any homeomorphism of the sphere which fixes three points is isotopic to the identity, via an isotopy fixing those three points. From this, it is easy to see that if $\#(X(g)) \leq 3$, $g$ is equivalent to a rational map.

If $\#(X(g))=4$ and $Q_{g}$ is not a hyperbolic orbifold, then there is only one possibility for the universal orbifold cover $\widetilde{Q}_{g}$ of $Q_{g}$ and for the covering map $\pi: \widetilde{Q}_{g} \rightarrow Q_{g}: \widetilde{Q}_{g}=\mathbf{C}, Q_{g}$ is the quotient of $\mathbf{C}$ by the relation $z \sim \pm z+m+n i$ $(m, n \in \mathbf{Z})$ and $\pi$ is the quotient map. Since $g^{-1}$ lifts to $Q_{g}$ and induces a homomorphism of a subgroup of the covering group onto the covering group (as always: see [T] 16.6) and the covering group in this case is of the form $\mathbf{Z}^{2} \times{ }_{s} \mathbf{Z}_{2}$, we see that $g^{-1}$ lifts injectively in this case, so that $g$ also lifts - to $\widetilde{g}: \mathbf{C} \rightarrow \mathbf{C}$, preserving equivalence classes in general, and those of critical orbits in particular. Hence, $g$ is equivalent to $[z] \mapsto[A z+\beta]$, for some real-linear map $A$ and $\beta \in(\mathbf{Z} / 2)^{2}$. In addition, $\operatorname{det}(A)=\operatorname{degree}(g)$. Let

$$
A=\left(\begin{array}{ll}
a & b \\
c & d
\end{array}\right)
$$

If $(a+d)^{2}<4(a d-b c), A$ is conjugate to a skew-symmetric matrix, and $g$ is equivalent to a rational map. Now let $a d-b c=2=\operatorname{degree}(g)$. If $(a+d)^{2}=9$, then the existence of an eigenvector of $A$ with eigenvalue \pm 1 implies the existence of a simple loop $\gamma$ in $\overline{\mathbf{C}} \backslash X(g)$ such that a component $\gamma^{\prime}$ of $g^{-1}(\gamma)$ is isotopic to $\gamma$ in $\overline{\mathbf{C}} \backslash X(g)$, and $g \mid \gamma^{\prime}$ is a homeomorphism. If $(a+d)^{2}>9$, then $A$ has eigenvalues $\lambda_{s}, \lambda_{u}$ with $\left|\lambda_{s}\right|<1<2<\left|\lambda_{u}\right|$.

\subsection{Levy Cycles.}

Let $g: \overline{\mathbf{C}} \rightarrow \overline{\mathbf{C}}$ be a critically finite degree two orientation-preserving branched covering. Then we recall that a set $\left\{\gamma_{1} \ldots \gamma_{r}\right\}$ of simple nontrivial disjoint loops in $\overline{\mathbf{C}} \backslash X(g)$ is a Levy cycle for $g$ if there exists $\gamma_{i}^{\prime}$ in $g^{-1}\left(\gamma_{i+1}\right)$ (writing $\gamma_{r+1}=\gamma_{1}$ ) such that $\gamma_{i}$ and $\gamma_{i}^{\prime}$ are isotopic in $\overline{\mathbf{C}} \backslash X(g)$ and $g \mid \gamma_{i}^{\prime}$ is a homeomorphism. We recall that Thurston's theorem for a necessary and sufficient condition for non-equivalence to a rational map [T] was given three equivalent formulations in the case of degree two in 1.6 of [R2] (where further references are given). The middle condition was the existence of a Levy cycle. In [R2] the hypothesis of Thurston's theorem was slightly simplified. In fact, the theorem holds whenever the associated orbifold is hyperbolic. Non-hyperbolic orbifolds have been investigated in 1.2 , and, putting these facts together, we 
see that, if $g$ is an orientation-preserving degree two critically finite branched covering, exactly one of the three properties stated in 1.1 does indeed hold.

So now we wish to obtain more information about $g$, where $g$ is a degree two critically finite orientation-preserving rational map with a Levy cycle. We start with two lemmas, which are, in fact, the basis of a proof of existence of a Levy cycle in the first place ([TL], for example). The proofs are included for convenience, since they are quite short.

1.4. Lemma. Let $g: \overline{\mathbf{C}} \rightarrow \overline{\mathbf{C}}$ be a critically finite degree two orientationpreserving branched covering. Let $\Gamma$ be a (finite) set of simple disjoint isotopically distinct loops in $\overline{\mathbf{C}} \backslash X(g)$ with $\Gamma \subset g^{-1} \Gamma$ up to isotopy. Let $\gamma \in \Gamma$ separate critical values. Then $\gamma$ bounds a disc $D$ such that all components of $g^{-n} D$ are discs, for all $n \geq 0$.

Proof:

Let $\Gamma_{1}$ be the set of loops $\gamma$ in $\Gamma$ such that $\gamma$ bounds a disc $D$ for which all components of $g^{-n} D$ are discs for all $n \geq 0$. Then $\Gamma \cap g^{-1} \Gamma \subset \Gamma_{1}$. (We mean "up to isotopy in $\overline{\mathbf{C}} \backslash X(g)$ ", but we omit saying this, for the rest of this proof.) Let $\Gamma_{2}=\Gamma \backslash \Gamma_{1}$. Then $\Gamma_{2} \subset g^{-1} \Gamma_{2}$. We claim that no loop in $\Gamma_{2}$ separates critical values. Otherwise, let $D$ be a disc containing exactly one critical value, bounded by a loop $\gamma$ from $\Gamma_{2}$, and containing no loop from $\Gamma_{2}$ in its interior. Then we can prove by induction on $n$ that all components of $g^{-n} D$ are discs, and none contains a loop from $\Gamma_{2}$ in its interior. For assume this is true for n, and $D_{1}$ is a disc component of $g^{-n} D$. Then $D_{1}$ cannot contain both critical values, since it does not contain $\gamma$ in its interior. So components of $g^{-1} D_{1}$ are all discs, and do not contain elements of $g^{-1} \Gamma_{2} \supset \Gamma_{2}$ in their interiors. The inductive step is completed. So $\gamma \in \Gamma_{1}$, giving a contradiction, and the lemma is proved.

\subsection{Degenerate Levy Cycles.}

A Levy cycle $\Gamma=\left\{\gamma_{1} \ldots \gamma_{r}\right\}$ for $g$ is degenerate if each $\gamma_{i}, \gamma_{i}^{\prime}$ (as in 1.3) bounds a disc $D_{i}, D_{i}^{\prime}$, with $D_{i}^{\prime}$ isotopic to $D_{i+1}$ via an isotopy preserving $X$, and $g: D_{i}^{\prime} \rightarrow D_{i}$ is a homeomorphism. Thus no disc $D_{i}$ contains a critical value of $g$. So if, for instance, the forward orbits of both critical points of $g$ contain periodic critical points, any Levy cycle for $g$ must be nondegenerate. The following lemma is then immediate.

\section{Lemma.}

Let $g$ be a critically finite degree two orientation-preserving branched covering, such that any Levy cycle is nondegenerate. Let $\gamma$ be a loop in $\overline{\mathbf{C}} \backslash X(g)$ bounding a disc $D$ such that all components of $g^{-n} D$ are discs for all $n \geq 0$. Then $D$ cannot contain any loop of a Levy cycle. 


\subsection{Complete Levy sets.}

Let $f: \overline{\mathbf{C}} \rightarrow \overline{\mathbf{C}}$ be a critically finite degree two orientation-preserving branched covering, and let $\Gamma$ be a finite set of disjoint simple nontrivial loops in $\overline{\mathbf{C}} \backslash X(f)$. Then $\Gamma$ is a complete Levy set (for $f$ ) if the conditions below hold, writing $X=X(f)$.

The Levy Condition. $\Gamma \subset f^{-1} \Gamma$, and for $\gamma \in f^{-1} \Gamma, \gamma \in \Gamma$ if and only if $\gamma$ does not bound a disc $D$ such that all components of $f^{-n} D$ are discs, for all $n$.

Thus, by Lemma 1.4, no loop in $\Gamma$ separates critical values, and $f$ is degree one restricted to any component of $f^{-1} \Gamma$. There are Levy cycles in $\Gamma$, but $\Gamma$ may not be a union of Levy cycles.

The Gap Invariance Condition. If $G$ is a component of $\overline{\mathbf{C}} \backslash \cup \Gamma$ such that $G \backslash X$ is not an annulus, then there is a unique component $G^{\prime}$ of $\overline{\mathbf{C}} \backslash X$ - which we term $f_{*} G$ - and a unique component $G^{\prime \prime}$ of $f^{-1} G^{\prime}$ such that $G^{\prime \prime} \subset G$ and $\partial G \subset \partial G^{\prime \prime}$.

Definition. $G$ is a gap for $\Gamma$ if $G$ is a component of $\overline{\mathbf{C}} \backslash \cup \Gamma$ such that $G \backslash X$ is not an annulus.

The Gap Restrictions. No annulus gap of $\overline{\mathbf{C}} \backslash(X \cup(\cup \Gamma))$ is mapped onto itself by $f^{n}$ (any $n>0$ ). If $G_{1}, G_{2}$ are distinct with $\bar{G}_{1} \cap \bar{G}_{2} \neq \phi$, then at least one $G_{i}$ is not mapped homeomorphically by all $f^{n}$. Any Levy cycle disjoint from $\Gamma$ can be isotoped in $\overline{\mathbf{C}} \backslash X$ either into $\Gamma$ itself or into a periodic cycle of gaps on which $f$ acts homeomorphically.

1.7. Lemma. Let $g$ have at least one nondegenerate Levy cycle, but no degenerate ones. Then there exists a complete Levy set $\Gamma$ for some $f$, where $f$ is homotopic to $g$ via a homotopy $\left\{f_{t}\right\}$ through critically finite branched coverings with $X\left(f_{t}\right)=X(g)$ for all $t$.

Proof: Let $\Gamma_{0}$ be a maximal union of disjoint Levy cycles for $g$. Let $k>0$ be such that, if $\gamma \in g^{-(k+1)} \Gamma_{0}$, and $\gamma$ does not bound a disc $D$ such that all components of $g^{-n} D$ are discs for all $n>0$, then $\gamma \in g^{-k} \Gamma_{0}$ up to isotopy. Let $\Gamma_{1}$ be a set of two copies, up to isotopy, of all such loops in $g^{-(k+1)} \Gamma_{0}$ (or, equivalently, in $g^{-k} \Gamma_{0}$ ). Then $g, \Gamma_{1}$ satisfy the Levy Condition except that $\Gamma_{1} \subset g^{-1} \Gamma_{1}$ up to isotopy only. Note that $\Gamma_{1}$ contains up to isotopy all loops in $g^{-1} \Gamma_{1}$ which separate two elements of $\Gamma_{1}$. Hence $G^{\prime \prime}, G^{\prime}$ exist as in the Gap Condition, up to isotopy. Then $f$ can be chosen equivalent to $g$ (via a homotopy preserving $X$ ) so that the Levy and Gap Conditions are satisfied by $f, \Gamma_{1}$. If $G_{1}, G_{2}$ are distinct gaps for $\Gamma_{1}$ such that $f^{n}$ always maps both of them homeomorphically, then if one of them is periodic they both are, and the period of both is $\leq$ the period of the common boundary component. So obtain $\Gamma$ from $\Gamma_{1}$ by removing all such common boundary components and all their preimages under all $f^{n}$, and also removing redundant loops in annuli (possibly removing some annuli gaps altogether) so that $\Gamma$ satisfies the Gap Restrictions. 
The last Gap Restriction is satisfied because $\Gamma_{0}$ was a maximal union of Levy cycles.

\subsection{Critical gaps for Complete Levy Sets.}

From now on, until the end of this chapter, let $f: \overline{\mathbf{C}} \rightarrow \overline{\mathbf{C}}$ be a critically finite degree two orientation-preserving branched covering with no degenerate Levy cycle, and let $\Gamma$ be a complete Levy set for $f$. Then by Lemma 1.4, and the Levy Condition, both critical values of $f$ are contained in the same gap for $\Gamma$. Hence, both critical points are contained in the same gap, which we call the critical gap, usually denoted by $G_{0}$. The following lemma holds, under the hypotheses of this section. It, too, can be found essentially in [TL].

Lemma. The disc gaps for $\Gamma$ are $f_{*}^{i} G_{0}$, with $1 \leq i \leq r$ for some $r \geq 2$.

Proof: There is a gap $G$ which is a disc. Then $G \cap X(f) \neq \phi$. So $f^{n} c \in G$ for some least $n \geq 1$ and $c$ critical. If $n>1$ then both components of $f^{-1} G$ are discs. Then if we write $G_{n}=G$, we can find $G_{i}(1 \leq i \leq n)$ with $f_{*} G_{i}=G_{i+1}$ for $i<n$ and $f^{i} c \in G_{i}$. Then $G_{i}$ is a disc for each $i$, and $G_{1}$ is the gap containing the critical values, as required. We must have $r \geq 2$, because $\overline{\mathbf{C}}$ has Euler characteristic 2, so at least two components of $\overline{\mathbf{C}} \backslash(\cup \Gamma)$ are discs.

\subsection{Branched coverings associated to gaps.}

Let $G$ be a gap for $\Gamma$ which is of period $n$ under $f_{*}$. (All gaps must be eventually periodic under $f_{*}$, since there are only finitely many gaps.) Let $G_{1}$ be the component of $f^{-n} G$ with $G_{1} \subset G, \partial G \subset \partial G_{1}$. Then $G_{1}=S G$ for an inverse branch $S$ of the branched covering $f^{n}$. Inductively we can define $G_{k+1}=S G_{k}$. Then for some least $k \geq 0$, all components of $\partial G_{k+1} \backslash \partial G_{k}$ are trivial in $\overline{\mathbf{C}} \backslash X(f)$.

Now $f^{n}$ maps components of $\partial G_{k+1}$ to components of $\partial G_{k}$, and maps components of $\partial G$ to components of $\partial G$. Also, $f^{n} \mid G_{k+1}$ is either a homeomorphism or a degree two branched covering, depending on whether or not the critical gap is in the forward orbit of $G$. Then we define $h=h_{G}$ by

a) $h=f^{n}$ on $G_{k+1}$,

b) $h(D)=D^{\prime}$ if $D, D^{\prime}$ are components of $\overline{\mathbf{C}} \backslash G_{k+1}, \overline{\mathbf{C}} \backslash G_{k}$ with $h(\partial D)=\partial D^{\prime}$,

c) each component of $\overline{\mathbf{C}} \backslash G_{k+1}$ contains at most one point of $h^{-1} X(h)$.

Then if $G$ is in the forward orbit of the critical gap, $h$ is the critical branched covering of $f$. It is uniquely defined up to equivalence (given $\Gamma$ ), and $h$ has no Levy cycle, since $\Gamma$ is a complete Levy set. (See the Gap Restrictions of 1.6.) If the critical gap is not in the forward orbit of $G$, then $k=1$ and $f^{n}=h: G \rightarrow G$ is a homeomorphism. 
1.10. Lemma. Let the critical gap $G_{0}$ of $\Gamma$ be periodic and the critical branched covering $h$ be not equivalent to a rational map. Then the period of $G_{0}$ is less than the period of any periodic critical point of $f$.

Proof: Let $n$ be the period of $G_{0}$. Let $G_{1}$ be the component of $f^{-2 n} G_{0}$ with $G_{1} \subset G_{0}, \partial G_{0} \subset \partial G_{1}$. Now by $1.2, h$ has no fixed critical points (in fact, no periodic critical points). Then for any critical point $c$, there is a disc component $D$ of $G_{0} \backslash G_{1}$ containing $c$ such that $f^{n} D$ is another component of $G_{0} \backslash G_{1}$ and $f^{i} D \cap G_{0}=\phi$ for $0<i<n$. So if $c$ is periodic, $c$ must have period $>n$.

\subsection{The Fixed Set $P$.}

By the Lefschetz Fixed Point Theorem, some loop or gap $P$ of $\Gamma$ is fixed by $f$. (This is the third equivalent condition of 1.6 of [R2] for nonequivalence to a rational map. This proof is due to Shishikura.) If $P$ is a loop, it must have orientation reversed, because otherwise one of the discs it bounds is fixed, contradicting the Levy Condition. Neither can $P$ be the critical gap, because if it is, any periodic boundary component is a degenerate Levy cycle for $f$. So if $P$ is a gap, $f: P \rightarrow P$ is a homeomorphism, and boundary components are cyclically permuted, again because there are no degenerate Levy cycles.

\subsection{2 .}

Now we can summarise the main homotopy-type information we shall use.

\section{Decomposition Proposition.}

Let $g: \overline{\mathbf{C}} \rightarrow \overline{\mathbf{C}}$ be an orientation-preserving critically finite degree two branched covering which is not equivalent to a rational map, and with no degenerate Levy cycle. Let $f$ be an equivalent branched covering (via a homotopy which is constant on $X(g)=X(f))$ with complete Levy set $\Gamma$, critical gap $G_{0}$ and fixed loop or gap $P$. Let $\ell$ be the number of boundary components of $P$, or $\ell=2$ if $P$ is a loop. Then one of the following holds.

Rational Critical Branched Covering. The critical gap $G_{0}$ is periodic un$\operatorname{der} f_{*}$, and the critical branched covering is equivalent to a rational map.

Periodic Separating Set. A gap $Q$ is mapped homeomorphically onto itself by $f^{\ell}, Q$ separates $P$ and $f_{*} G_{0}$, and $\bar{Q} \cap \bar{P}=\bar{Q} \cap \overline{f_{*} G_{0}}=\phi$.

Adjacent Periodic Set. The gap $f_{*} G_{0}$ is periodic, and adjacent to some periodic gap or loop of lesser period than itself. 


\subsection{Outline of proof of the Decomposition Proposition.}

Now we fix some notation. Let $\Gamma$ be a complete Levy set for $f$, and let $P$, $\ell$ be as in the statement of the proposition. Let $R$ be the set bounded by $P$ and $f_{*} G_{0}$ - which is either a closed annulus or a loop of $\Gamma$. Clearly, if $R$ is a loop of $\Gamma, G_{0}$ has period $\ell$, and the converse is also true, because no annulus component of $\overline{\mathbf{C}} \backslash \cup \Gamma$ can be mapped homeomorphically to itself by $f^{\ell}$, by the definition of complete Levy set. Let $\gamma=\partial R \cap \partial P$. Let $T$ be the local inverse of $f^{\ell}$ defined on $R$ such that $T$ preserves $\gamma$. Let $S$ be the local inverse of $f^{\ell}$ defined on $R$ which is $\neq T$ and such that $S R$ is in the same component of $\overline{\mathbf{C}} \backslash P$ as $f_{*} G_{0}$ and $R$. Now we prove the following lemma, under the assumptions that $R$ is a closed annulus and $G_{0}$ does not have period $\ell$. It clearly completes the proof of the Decomposition Proposition.

1.14. Lemma. $\quad T R \subset R, S R \cap R \neq \phi$, and there is a set $Q$ in $S R \cap R$ which is either a gap or loop of $\Gamma$, such that $Q$ has period $\ell$ under $f$ and is mapped homeomorphically by $f^{\ell}$, and $Q$ separates $f_{*} G_{0}$ from $T R$.

We split the proof of this lemma itself into a number of stages.

1.15. Lemma. There are least integers $N, N^{\prime} \geq 0$ with

$$
f_{*}^{N \ell+1} G_{0} \cap S R=\phi, f_{*}^{N^{\prime} \ell+1} G_{0} \cap T R=\phi .
$$

Proof: The property derived from the definition of complete Levy set that we use repeatedly is:

(1) for all $i \geq 0, f_{*}^{i} G_{0}$ is the connected union of a component of $f^{-1} f_{*}^{i+1} G_{0}$ and some (possibly none) discs which are disjoint from $\cup \Gamma$.

If $N$ does not exist (for example) then $f_{*}^{i} G_{0}$ is in the component of $\overline{\mathbf{C}} \backslash P$ bounded by $f^{i-1} \gamma$ for all $i$. (If $N$ does exist, this is true for $i \leq N \ell$.) If $G_{0}$ is periodic, this implies $G_{0}=f_{*}^{p \ell} G_{0}$ for some $p \geq 2$. But $G_{0} \cap f^{-1} R=\phi$, so then $f_{*}^{(p-1) \ell+1} G_{0} \cap S R=\phi$, and $N$ exists. If $G_{0}$ is not periodic, there are integers $i, j \geq 1$ such that $f_{*}^{i \ell+1} G_{0}=f_{*}^{j \ell+1} G_{0}$ but $f_{*}^{i \ell} G_{0}, f_{*}^{j \ell} G_{0}$ are distinct and separated by $G_{0}$. Then $f_{*}^{i \ell} G_{0}, f_{*}^{j \ell} G_{0}$ cannot intersect the same component of $f^{-1} R$. Then $f_{*}^{(i-1) \ell+1} G_{0}$ or $f_{*}^{(j-1) \ell+1} G_{0}$ is disjoint from $S R$, and, again, $N$ exists. The proof of the existence of $N^{\prime}$ is exactly similar.

Now $f_{*}^{i} G_{0} \neq f_{*}^{N \ell+1} G_{0}$ for $1<i<N \ell+1$, so $f_{*}^{i} G_{0}(1<i \leq N \ell+1)$ are all distinct. Hence they are all disjoint, and disjoint from $P$ for $0 \leq i<N \ell+1$. A similar statement holds with $N$ replaced by $N^{\prime}$.

1.16. Lemma. $N^{\prime}=0$, so that $T R \subset R$ and $S R \cap R \neq \phi$, and $S \partial f_{*} G_{0}$ separates $f_{*} G_{0}$ from $P$. 
Proof: It follows from 1.15 that

(1) $f_{*}^{i \ell+1} G_{0}$ is the union of $T f_{*}^{(i+1) \ell+1} G_{0}$ and some discs disjoint from $X(f)$, for $0 \leq i \leq N^{\prime}-2$.

Let $R_{0}^{\prime}=R$. Inductively, define $R_{i+1}^{\prime}=T R_{i}^{\prime} \backslash f_{*} G_{0}$. Then $R_{i}^{\prime} \neq \phi$ for all $i$ by induction, since $\gamma \subset T R_{i}^{\prime}$, and $R_{i+1}^{\prime} \subset R_{i}^{\prime}$. But by 1.15, using (1),

$$
T R_{j}^{\prime} \cap f_{*}^{i \ell+1} G_{0} \neq \phi \text {, but } T R_{j}^{\prime} \cap f_{*}^{\left(N^{\prime}-j\right) \ell+1} G_{0}=\phi
$$

for $0 \leq j \leq N^{\prime}$, and $0 \leq i<N^{\prime}-j$. In particular, $T R_{i}^{\prime} \cap f_{*} G_{0} \neq \phi$ for $0 \leq i<N^{\prime}$, but $T R_{N^{\prime}}^{\prime} \cap f_{*} G_{0}=\phi$, so $R_{N^{\prime}+1}^{\prime}=T R_{N^{\prime}}^{\prime}$, and

$$
\begin{gathered}
\partial R_{j}^{\prime}=\gamma \cup \bigcup_{i=0}^{j} T^{i} \partial f_{*} G_{0} \text { for } j \leq N^{\prime}, \\
\partial R_{N^{\prime}+1}^{\prime}=\gamma \cup \bigcup_{i=1}^{N^{\prime}+1} T^{i} \partial f_{*} G_{0} .
\end{gathered}
$$

See Diagram 1. The shaded region denotes $T R_{N^{\prime}}$ (drawn in the case $N^{\prime}=1$, which does not actually happen).

\section{Diagram 1}

Then $T^{N^{\prime}+1} \partial f_{*} G_{0}$ must bound a disc disjoint from $R_{N^{\prime}+1}^{\prime}=T R_{N^{\prime}}^{\prime}$ containing $\partial f_{*} G_{0}$. Then all boundary components of $T^{k} R_{N^{\prime}}^{\prime}$ are nontrivial in $\overline{\mathbf{C}} \backslash X$ for $k \geq 0, T^{k+1} R_{N^{\prime}}^{\prime} \subset T^{k} R_{N^{\prime}}^{\prime}$ and for some $p \geq 0, T^{p+1} R_{N^{\prime}}^{\prime}$ and $T^{p} R_{N^{\prime}}^{\prime}$ are 
isotopic in $\overline{\mathbf{C}} \backslash X(g)$. If $N^{\prime}>0$ then $T^{p} R_{N^{\prime}}^{\prime}$ has $N^{\prime}+2 \geq 3$ boundary components, all in the full orbit of $\partial R$. Since $\Gamma$ is a complete Levy set, $\partial T^{p} R_{N^{\prime}}^{\prime} \subset \Gamma$, and there is a gap $G$ for $\Gamma$ isotopic to $T^{p} R_{N^{\prime}}^{\prime}$. But then $\bar{G} \cap \bar{P} \neq \phi$ with $f_{*}^{n} G \neq G_{0}$ for any $n \geq 0$, which is a contradiction to the definition of a complete Levy set. So $N^{\prime}=0$. In particular, $T R \subset R$. Then $T R$ and $S R$ are separated by a component of $f^{-\ell} f_{*} G_{0}$ which is disjoint from $f_{*} G_{0}$. So $S R \cap R \neq \phi$, and $S \partial\left(f_{*} G_{0}\right)$ separates $f_{*} G_{0}$ from $P$.

\subsection{Proof of Lemma 1.14.}

Let $R=R_{0}$, and let $R_{1}$ be the annulus bounded by $S \partial\left(f_{*} G_{0}\right)$ and $\partial f_{*} G_{0}$, so that $R_{1} \subset R=\operatorname{domain}(S)$, and $R_{1}=R \cap S R$. Then define

$$
R_{i+1}=S R_{i} \backslash f_{*} G_{0} .
$$

As in 1.16 , we see that

$$
S R_{j} \cap f_{*}^{i \ell+1} G_{0} \neq \phi \text {, but } S R_{j} \cap f_{*}^{(N-j) \ell+1} G_{0}=\phi
$$

for $0 \leq j \leq N$ and $0 \leq i<N-j$. Hence, $R_{j} \neq \phi$ for $0 \leq j \leq N+1$, $R_{N+1}=S R_{N}$. Then if $N \geq 1$,

$$
\begin{gathered}
\partial R_{j}=\cup_{i=0}^{j} S^{i} \partial G_{0} \text { for } j \leq N \\
\partial R_{N+1}=\cup_{i=1}^{N+1} S^{i} \partial G_{0} .
\end{gathered}
$$

If $N=0, \partial R_{1}=S \partial R$. Proceeding as in the case of $T R$, we obtain $Q$. If $N \geq 2, Q$ is isotopic to $S^{p} R_{N}$ for some $p \geq 0$ and is a gap for $\Gamma$ of period $\ell$ with boundary components cyclically permuted. If $N=0$ or $1, Q$ is isotopic to $\partial S^{p} R_{N}$ for $p \geq 0, Q$ is part of a Levy cycle of period $\ell$ and has oriented period $2 \ell$, up to isotopy. So $Q$ has the required properties.

1.18 Note. Tan Lei's proof of the existence of a minimal Levy cycle [TL] (that is, the existence of $P$ in the current language) was obtained by taking the least integer $n>1$ with $f_{*}^{n} G_{0} \cap P^{\prime}=\phi$, where $P^{\prime}$ is the annulus separating $f_{*} G_{0}$ and $f^{-1} f_{*} G_{0}$, and then taking successive inverse images under $f^{n}$ of $P^{\prime} \backslash \cup_{i<n} f_{*}^{i} G_{0}$. The proof above was obtained by generalising this idea.

\subsection{Examples.}

It might be as well to give some examples to show that the various alternatives of the Decomposition proposition are all quite common. We use matings to obtain the examples, and refer to 1.19 of [R2] for the notations. So let $p, q$ be odd denominator rationals. Then $f=s_{p} \amalg s_{q}: \overline{\mathbf{C}} \rightarrow \overline{\mathbf{C}}$ is a type IV branched covering which preserves a lamination $L_{p} \cup L_{q}^{-1}$. If $f$ is not a rational map, then some leaves of $L_{p} \cup L_{q}^{-1}$ join up to form closed loops. 
Example 1. If $p=q=1 / 3$, the two leaves joining the points $1 / 3,2 / 3$ join to give a loop which is, in itself, a Levy cycle, with the critical gap adjacent to it. We can easily identify the critical branched covering by examining $\left(s_{p} \amalg s_{q}\right)^{2}$ restricted to the union of the gaps containing $0, \infty$, and we see that the critical branched covering is equivalent to $z \mapsto z^{2}$. This gives the Rational Critical Branched Covering alternative of the Decomposition Proposition.

Example 2. If $p$ and $1-q$ are separated from 0 by a finite-sided gap of $Q M L$ such as the triangle with vertices at $9 / 56,11 / 56,15 / 56$ - but $p$ and $1-q$ are not in the same component of the finite-sided gap complement, then the critical branched covering is a homeomorphism.

Example 3. Let $p, q$ be on minor leaves which are separated from 0 by the leaf with endpoints $\frac{17}{48}, \frac{19}{48}$. See Diagram 2.

\section{Diagram 2}

As in example 1, we can identify the critical branched covering by examining $\left(s_{p} \amalg s_{q}\right)^{2}$ restricted to the union of the gaps containing $0, \infty$, and in this case, the critical points $c_{1}, c_{2}$ of the critical branched covering $h$ satisfy $h\left(c_{1}\right) \neq h\left(c_{2}\right)$, but $h^{2}\left(c_{1}\right)=h^{2}\left(c_{2}\right)$, and $h^{3}\left(c_{i}\right) \neq h^{2}\left(c_{i}\right)$ but $h^{4}\left(c_{i}\right)=h^{3}\left(c_{i}\right)$. Then $h$ is actually equivalent to a rational map. But we can modify this example as follows. First, perturb $s_{p} \amalg s_{q}$ to $g$ having a complete Levy cycle, so that the critical gap $G$ contains a four-holed sphere component $G^{\prime}$ of $g^{-2} G$ not intersecting $X(g)$. Then we can change $g$ to another branched covering $g_{1}$ with $X(g)=X\left(g_{1}\right)$ by changing the definition only on $G^{\prime} \cup g G^{\prime}$. Then we can choose $g_{1}$ so that the new 
critical branched covering is equivalent to $[x] \mapsto[A x]: T^{2} / \sim \rightarrow T^{2} / \sim$, where $A$ is any integral matrix with determinant 2 and all matrix entries odd, to ensure that the critical orbits have the same pattern as for $h$. 


\section{Chapter 2. The Fixed Holed-Sphere Theorem.}

2.1.

In this chapter we prove a semiconjugacy result. We recall that there are many instances in dynamical sytems of homotopy information (usually containing some sort of "expanding" or "hyperbolic" criterion) for a map $g$ yielding semiconjugacy information, that is, the existence of a nontrivial map $g_{1}$ and continuous $\varphi$ such that $\varphi \circ g=g_{1} \circ \varphi$. The reference which comes to mind, and which is actually relevant here, is [Fr]. There it is proved (for example) that if $T^{2}$ is the two-torus, and $g: T^{2} \rightarrow T^{2}$ is continuous and homotopic to a linear automorphism $g_{1}$ of $T^{2}$ with both eigenvalues off the unit circle, then a $\varphi$ homotopic to the identity exists. The same result holds - and can be proved more simply - if $g_{1}$ is, instead, an expanding linear endomorphism. However, somewhat surprisingly, the result does not hold if $g_{1}$ is simply an endomorphism, even if both eigenvalues are off the unit circle. We can see this as follows. Let $g_{1}$ be such that the associated matrix $A$ is in $G L(2, \mathbf{Z})$ and has determinant 2 and eigenvalues $\lambda_{u}, \lambda_{s}$ with $\left|\lambda_{s}\right|<1<2<\left|\lambda_{u}\right|$, with corresponding eigenvectors $v_{u}, v_{s}$. Identify the two-torus with $\mathbf{R}^{2} / \mathbf{Z}^{2}$. We are now going to construct a covering map $g: T^{2} \rightarrow T^{2}$ by constructing the well-defined lift, $\bar{g}$, of the multivalued function $g^{-1}$ to $\mathbf{R}^{2}$ (so that notation agrees with what we shall do later). In fact, we can take $\bar{g}$ to be an arbitrarily small $C^{0}$ perturbation of $A^{-1}$. We choose $\bar{g}$ so that $\bar{g}(0,0)=(0,0)$, and for some $(m, n) \in \mathbf{Z}^{2}$, $(m, n) \neq(0,0)$, the set

$$
\left\{(x, y): \lim _{k \rightarrow \infty} \bar{g}^{k}(x, y)=(0,0)\right\}
$$

contains $(m, n)$. Note that the set

$$
\left\{(x, y): \lim _{k \rightarrow \infty} A^{-k}(x, y)=(0,0)\right\}
$$

is simply $\operatorname{sp}\left(v_{u}\right)$, hence is not rationally defined and does not contain $(m, n)$. Then if Franks' theorem held for $g$, there would exist $\Psi: \mathbf{R}^{2} \rightarrow \mathbf{R}^{2}$ satisfying

a) $\Psi((x, y)+(p, q))=\Psi(x, y)+(p, q)$ for all $(x, y) \in \mathbf{R}^{2}$ and all $(p, q) \in \mathbf{Z}^{2}$, b) $\Psi \circ \bar{g}=A^{-1} \circ \Psi$.

Note that b) yields $\Psi(0,0)=(0,0)$. Then $\Psi\left(\bar{g}^{k}(m, n)\right)=A^{-k}(m, n) \rightarrow 0$ as $k \rightarrow \infty$, giving a contradiction to the existence of $\Psi$. Despite the fact that Franks' theorem does not hold in such circumstances, the technique of the proof can still be used to obtain weaker results. We shall do this in the case we are interested in, namely, branched coverings of $\overline{\mathbf{C}}$. Recall that, in Chapter 1 , torus endomorphisms came up in the description of branched coverings of $\overline{\mathbf{C}}$. This is the reason for the rather involved statement of the Fixed HoledSphere Theorem below. Note that the theorem has two alternative conclusions. Awkward torus maps (such as the one above) are more involved in the second 
conclusion. The theorem can be applied to branched coverings which are also lamination maps. For the definitions of invariant laminations and lamination maps, and their purpose, see the extended introduction of [R2].

2.2. The Fixed Holed-Sphere Theorem. Let $g: \overline{\mathbf{C}} \rightarrow \overline{\mathbf{C}}$ be an orientationpreserving critically finite degree two branched covering, with at least one nondegenerate Levy cycle, and no degenerate Levy cycle. In a neighbourhood of any periodic critical point in $X=X(g)$ fixed by $g^{p}$, let there exist a local topological conjugacy to $z \mapsto z^{n}$ for suitable $n$.

Then there is an open subset $Y$ of $\overline{\mathbf{C}} \backslash X$, and an integer $m$, such that $\overline{\mathbf{C}} \backslash Y$ has $\ell \geq 2$ components, each intersecting $X, g^{m} \mid \bar{Y}$ is a homeomorphism, $Y$ and $g^{m} Y$ are isotopic in $\overline{\mathbf{C}} \backslash X$, with $g^{m}$ cyclically permuting the boundary components, and one of the following holds.

Expanding Fixed Set Case : $m=1$ and $\bar{Y} \subset g Y$.

Nonrational Adjacent Case: $m \ell$ is less than the period of any point in $X$ and $\partial Y=\partial_{1} \cup \partial_{2}$, where $\partial_{1}$ and $\partial_{2}$ have the following properties. Each $\partial_{i}$ is a finite disjoint union of closed arcs, two on each component of $\partial Y, \partial_{1} \cap \partial_{2}$ is the set of endpoints of these arcs, and $\partial Y \cap g^{m} \bar{Y} \subset \partial_{1}, \partial g^{m} Y \cap \bar{Y} \subset g^{m} \partial_{2}$. Each component of $Y \backslash g^{m} Y$ has one component of intersection with each of $\partial_{1}$ and $g^{m} \partial_{1}$, and 2 components of intersection with $\partial_{2}$. Each component of $g^{m} Y \backslash Y$ has one component of intersection with each of $\partial_{2}$ and $g^{m} \partial_{2}$, and 2 components of intersection with $g^{m} \partial_{1}$.

See Diagram 3 for what $Y$ looks like in the nonrational adjacent case.

\section{Diagram 3}




\subsection{Different Cases of, and Additions to, the Fixed Holed-Sphere Theorem.}

The different cases of the Fixed Holed-Sphere Theorem are dependant on the different conclusions of the Decomposition Proposition of Chapter 1. All the proofs start with the same basic idea, but then develop differently. Let $f$ be the branched covering of the Decomposition Proposition which is equivalent to $g$ via a homotopy $\left\{f_{t}\right\}$, such that $f$ has a complete Levy set $\Gamma$, fixed loop or gap $P$. The Expanding Fixed Set Case of the Fixed Holed-Sphere Theorem holds when either of the first two cases of the Decomposition Proposition hold, that is, the Rational Critical Branched Covering Case, or the Separating Periodic Set Case. The Nonrational Adjacent Case of the Fixed Holed Sphere Theorem holds when the critical branched covering of $f$ is nonrational and the Adjacent Periodic Set Case of the Decomposition Theorem holds.

Some unfortunately complicated variations on the Fixed Holed Sphere Theorem will be needed later, when we come to apply this result to lamination maps. We state these now.

Variation in the Fixed Set Case. If the condition $\bar{Y} \subset g Y$ is weakened to $\bar{Y} \subset g^{n} Y$ for somen, then, given $M>0$, we can ensure that, for any arc $\gamma$ such that $g^{k} \gamma$ is of length $\leq M$, for all sufficiently large $k$, then $\gamma \subset Y$.

Variations in the Nonrational Adjacent Case.

1. Given an integer $p$, we can ensure that $g^{i m}\left(g^{m} Y \backslash Y\right) \cap Y=\phi$ for $0 \leq i \leq p$.

2. Given $M^{\prime \prime}>0, Y$ can be chosen so that the length of any path in $Y \backslash g^{m} Y$ joining $\partial_{2}$ and $g^{m} \partial_{2}$ is $>M^{\prime \prime}$.

3. If $g$ is a lamination map preserving a lamination $L$, and $I_{i}(1 \leq i \leq r)$ is any finite collection of $S^{1}$-segments between noncompact leaves in gap polygons, then $\partial_{1} \cup \partial_{2}$ can be chosen to be a union of leaf segments and $S^{1}$-segments not including any $I_{i}$.

\subsection{Outline of proof of the Fixed Holed-Sphere Theorem.}

We can assume that the homotopy $f_{t}$ from $g$ to $f$ satisfies $f_{t}=g$ on a neighbourhood $U$ of the forward orbits of periodic critical points. Then as in the semiconjugacy proof of Chapter 4 of [R2], we can find homeomorphisms $\Psi_{t}$ $(t \in[0,1])$ with

$$
\begin{gathered}
\Psi_{0}=\text { identity, } \\
\Psi_{t}=\text { identity on } U \cup X, \\
f_{t} \circ \Psi_{t}=g .
\end{gathered}
$$

Then $f \circ \Psi_{1}=g$, and $\Psi_{1}\left(g^{-1} U\right)=f^{-1} U$. Then define $\Psi_{t+n}(n \geq 1)$ by

$$
\begin{gathered}
f^{n} \circ \Psi_{t+n}=\Psi_{t} \circ g^{n}, \\
\Psi_{t+n}=\text { identity on } U \cup X .
\end{gathered}
$$


Then $\Psi_{t+n+1}=\Psi_{t+n}$ on $g^{-n} U$ for $t \in[0,1]$. We also have

$$
f \circ \Psi_{n+1}=\Psi_{n} \circ g
$$

since $f^{n} \circ f \circ \Psi_{n+1}=f^{n+1} \circ \Psi_{n+1}=g^{n+1}=g^{n} \circ g=f^{n} \circ \Psi_{n} \circ g$. Our set $Y$ in the Rational Critical Branched Covering and Periodic Separating Set Cases will be obtained from sets of the form $\Psi_{n}(Z)$, where $Z$ is a neighbourhood of $P$.

\subsection{Proof of the Fixed Holed-Sphere Theorem: Rational Critical Branched Covering Case.}

This is the most satisfactory case to deal with. We define a continuous semimetric $d$ on $\overline{\mathbf{C}}$ with the following properties.

1. There is $\delta_{0}>0$ such that, given $\delta_{1}>0$, there are $C$ and $\lambda>1$ such that:

if $d\left(f^{i} x, f^{i} y\right)<\delta_{0}$ for $0 \leq i<n$ and $d\left(f^{i} x, X\right)>\delta_{1}$ for $0 \leq i<n$ then $d\left(f^{n} x, f^{n} y\right) \geq C \lambda^{n} d(x, y)$.

Note, however, that we might have $d(x, y)=0$ and $d(f x, f y)>0$.

2. If a set $A=\left\{x: d\left(x, x_{0}\right)=0\right\}$ is noncontractible, then it is mapped by $f$ to a set of the form $\left\{y: d\left(y, y_{0}\right)=0\right\}=B$. If $A$ is contractible then $\partial A$ is mapped into $\partial B$ for some such set $B$.

3. $d(x, y)=0$ for all $x, y \in P$. If $\varepsilon>0$ is sufficiently small, then $\{x: d(x, P)<$ $\varepsilon\}$ can be homotoped in $\overline{\mathbf{C}} \backslash X$ into an arbitrarily small neighbourhood of $P$.

4. $\{y: d(x, y)<\varepsilon\}$ is always connected.

Suppose this semimetric has been defined. Then as in Chapter 4 of [R2] (that is, using property 1 above) we obtain from the equation $f^{n} \circ \Psi_{t+n}=\Psi_{t} \circ g^{n}$ that

$$
d\left(\Psi_{n+1}(x), \Psi_{n}(x)\right) \leq C_{1} \lambda^{-n},
$$

for all $x$ and for some $C_{1}>0$ and $\lambda>1$. We use the fact that $\Psi_{n}=\Psi_{n+1}$ on $g^{-n} U$. Similarly, if $\gamma$ is such that $g^{n} \gamma$ always has length $\leq M$, then $\lim _{n \rightarrow \infty}$ diameter $\Psi_{n}(\gamma)=0$. (See the variations on the Fixed Holed Sphere Theorem.) Then we can find $\delta_{2}>0, N$ and $\mu>1$ such that, $d(X, P)>2 \delta_{2}$, and if

$$
d_{N}(x, y)=\sum_{i=0}^{N} d\left(f^{i} x, f^{i} y\right),
$$

and $d_{N}(x, y)<\delta_{2}, d(x, X)>\delta_{0}$, then $d_{N}(f x, f y) \geq \mu d_{N}(x, y)$. Then for $0<\varepsilon<\delta_{0}$, consider

$$
Y^{\prime}=\left\{x: \lim _{n \rightarrow \infty} d_{N}\left(\Psi_{n}(x), P\right)<\varepsilon\right\},
$$

which is automatically open. Then since, if $Z=\left\{z: d_{N}(z, P)<\varepsilon\right\}, \bar{Z} \subset f Z$, and $f \mid Z$ is a homeomorphism for $\varepsilon$ small enough, $\bar{Y}^{\prime} \subset g Y^{\prime}$ and $g \mid Y^{\prime}$ is a homeomorphism. Let

$$
Y=\operatorname{Interior}\left(Y^{\prime} \cup\left(\text { components of } \overline{\mathbf{C}} \backslash Y^{\prime} \text { disjoint from } X\right)\right) \text {. }
$$


Then $Y$ has the required properties.

We can, in fact, do much more than construct $d$ : we can construct a semiconjugacy $\pi$ between $g$ and $[f]$ on a certain subset of $\overline{\mathbf{C}}$, with dense image under $\pi$, where $[f]$ is as follows. Let $\sim$ denote the equivalence relation $x_{1} \sim x_{2}$ if and only if $d\left(x_{1}, x_{2}\right)=0$. Let $\pi: \overline{\mathbf{C}} \rightarrow \overline{\mathbf{C}} / \sim$ be the quotient map. Let $[f]([x])=[f(x)]$ if $f([x])=[f(x)]$ (where $[x]$ is the equivalence class of $[x]$ ), and in general $[f]([x])=\left[f\left(x^{\prime}\right)\right]$ for any $x^{\prime} \in \partial[x]$. By property 2 of $d,[f]$ is well-defined. Then $\pi \circ f=[f] \circ \pi$ on a subset of $\overline{\mathbf{C}}$. It follows that $\pi \circ \Psi_{n} \circ g=[f] \circ \pi \circ \Psi_{n+1}$ on a subset $A_{n}$ of $\overline{\mathbf{C}}$. Then we can show that $\pi \circ \Psi_{n}$ converges to a map $[\Psi]$ satisfying $[\Psi] \circ g=[f] \circ[\Psi]$ on $\lim \sup _{n \rightarrow \infty} A_{n}$.

\subsection{Construction of the semi-metric.}

Recall that the critical gap (see 1.8) $G_{0}$ is periodic under $f_{*}$, and that the critical branched covering is equivalent to a rational map, since we are in the Rational Critical Branched Covering case. Let us call the critical branched covering $h_{0}$. The critical points of $h_{0}$ may not be periodic. But the forward orbit of any periodic critical point is in $G_{0}$. We originally chose $h_{0}$ (in 1.8) with $h_{0}^{-1} G_{0} \subset G_{0}, \partial G_{0} \subset \partial h_{0}^{-1} G_{0}$. This is consistent with choosing $h_{0}$ so that any periodic critical point is locally conjugate to $z \mapsto z^{p}$ for suitable $p$, as we now do. We can also assume that $h_{0}=f^{n} \mid f^{-n} G_{0} \cap G_{0}$, if $G_{0}$ has period $n$. Now by the Semiconjugacy Proposition of 4.1 of [R2], if $h_{1}$ is the rational map equivalent to $h_{0}$, there is a semiconjugacy $\psi: \overline{\mathbf{C}} \rightarrow \overline{\mathbf{C}}$ with $\psi \circ h_{0}=h_{1} \circ \psi$, and $\psi$ must collapse each component of $\overline{\mathbf{C}} \backslash G_{0}$ to a point. (We do not really need the Semiconjugacy Proposition. We could just be more careful in the choice of $h_{0}$, so that it would be clear that $h_{1}$ existed.) Let $d_{s}$ denote the spherical metric on $\overline{\mathbf{C}}$, and define

$$
d_{G_{0}}(x, y)=d_{s}(\psi x, \psi y) .
$$

Then $d_{G_{0}}$ satisfies property 1 required for $d$.

Now let $G$ be any component of $\cup_{n \geq 0} f^{-n} G_{0}$ which is not contained in a disc $D$ such that all components of $f^{-m} D$ are discs for all $m \geq 0$. Let $\mathcal{G}_{n}$ be the set of those components $G$ for which $n$ is the least integer $\geq 0$ with $f^{n} G=G_{0}$. Then for $G \in \mathcal{G}_{n}$, define

$$
d_{G}(x, y)=\left(1 / 3^{n}\right) d_{G_{0}}\left(f^{n} x, f^{n} y\right) .
$$

Note that, from the definition of $\mathcal{G}_{n}, f^{n}: G \rightarrow G_{0}$ is a homeomorphism for $G \in \mathcal{G}_{n}$.

Now define $d$ as follows. For any $x, y \in \overline{\mathbf{C}}$, choose a path $\alpha:[0,1] \rightarrow \overline{\mathbf{C}}$ with $\alpha(0)=x, \alpha(1)=y$, and such that $\alpha$ has at most one component of intersection with any $G \in \cup_{n \geq 0} \mathcal{G}_{n}$. Then write

$$
\alpha^{-1}(G)=\left(s_{G}, t_{G}\right)
$$


if $\alpha^{-1}(G) \neq \phi$. Then define

$$
d(x, y)=\sum_{\alpha^{-1}(G) \neq \phi} d_{G}\left(\alpha\left(s_{G}\right), \alpha\left(t_{G}\right)\right) .
$$

Then $d$ is well-defined and finite, since $\#\left(\mathcal{G}_{n}\right) \leq 2^{n}$, so that

$$
\sum_{n=0}^{\infty} \sum_{G \in \mathcal{G}_{n}}\left(1 / 3^{n}\right)<\infty
$$

It is then easy to check that $d$ has the required properties.

\subsection{Proof of the Fixed Holed-Sphere Theorem: A preliminary lemma in the Periodic Separating Set Case.}

In this case, $Q$ exists of period $\ell$ under $f_{*}$, separating $P$ from $f_{*} G_{0}$. See 1.14 for the following. There is a local inverse $T$ of $f^{\ell}$ defined on $P \cup R$, where $R$ is the set bounded by $f_{*} G_{0}$ and $P$, with $T P=P$ and $T R \subset R$. There is a local inverse $S \neq T$ of $f^{\ell}$ defined on a neighbourhood of $Q$ with $S Q=Q$, such that $S$ either reverses orientation on $Q$, if $Q$ is a loop, or cyclically permutes the boundary components of $Q$ (if $Q$ is a gap) and $S^{k}$ fixes them, for some $k \geq 3$. Thus, $T Q$ separates $P$ and $Q$, and $T\left(\partial R \cap \partial f_{*} G_{0}\right)$ - in the boundary of a component $G_{1}$ of $\cup_{n>0} f^{-n} G_{0}$ - separates $Q$ and $T Q$.

Lemma. There are $G_{i}, G_{i}^{\prime}(i \geq 1)$ between $Q$ and $T Q$, such that a) $G_{i}^{\prime}$ separates $G_{j}$ from $T Q$ for all $i, j$,

b) $G_{i}$ separates $G_{j}$ from $Q$, and $G_{i}^{\prime}$ separates $G_{j}^{\prime}$ from $T Q$, if $i>j$,

c) there is $N_{1}$ such that

$$
G_{i}, G_{i}^{\prime} \subset \bigcup_{j \leq N_{1}+i \ell k} f^{-j} G_{0} .
$$

See Diagram 4.

Proof: $S^{p} G_{1}$ separates $Q$ and $f_{*} G_{0}$ for some $1 \leq p<k$. Then we can take $G_{i}=S^{k(i-1)} G_{1}$ and $G_{i}^{\prime}=T S^{p+k(i-1)} G_{1}$. Thus, $G_{i}, G_{i}^{\prime}$ are in the disjoint sets $S R, T R$ respectively, and have all the required properties.

\subsection{Proof of the Fixed holed Sphere Theorem in the Periodic Sepa- rating Set Case.}

Let $Z_{0, i}, Z_{1} Z_{2, i}, Z_{3}, Z_{4, i} Z_{5, i}, Z_{6}$ be the open sets containing $P$ and bounded by the images under $f^{j}(0 \leq j<\ell)$ of $G_{i}^{\prime}, T Q, T G_{i}, T^{2} Q, T^{2} G_{i}$, $T^{2} G_{i}^{\prime} T^{3} Q$ respectively. Then

$$
\bar{Z}_{6} \subset Z_{5, i+1} \subset Z_{5, i} \subset \bar{Z}_{5, i} \subset Z_{4, i} \subset \bar{Z}_{4, i} \subset Z_{4, i+1} \subset Z_{3} \subset \bar{Z}_{3} \subset Z_{2, i} \subset \bar{Z}_{2, i}
$$




$$
\begin{gathered}
\text { Diagram } 4 \\
\subset Z_{2, i+1} \subset Z_{1} \subset \bar{Z}_{1} \subset Z_{0, i+1} \subset \bar{Z}_{0, i+1} \subset Z_{0, i}
\end{gathered}
$$

We take

$$
Y_{j, i, n}=\Psi_{n}^{-1} Z_{j, i}
$$

if $j=0,2,4$ or 5 , and

$$
Y_{j}=\left\{z: \overline{\left\{\Psi_{n}(z): n \geq N\right\}} \subset Z_{j} \text { for some } N\right\}
$$

if $j=1,3$ or 6 . We shall find $N_{2}$ such that, if $n, n^{\prime} \geq N_{2}+i \ell k$, for any $i$,

$$
Y_{6} \subset \bar{Y}_{6} \subset Y_{5, i, n} \subset Y_{4, i, n} \subset \bar{Y}_{4, i, n} \subset Y_{1} \subset \bar{Y}_{1} \subset Y_{0, i, n^{\prime}} .
$$

Then we take $Y$ to be the union of the component of Interior $\left(Y_{6}\right)$ which is nontrivial in $\overline{\mathbf{C}} \backslash X$, together with all complementary components which do not intersect $X$. Then $g^{2 \ell} Y$ is the component of Interior $\left(Y_{1}\right)$ which is nontrivial in $\overline{\mathbf{C}} \backslash X$, together with all complementary components which do not intersect $X$. Since $f^{2 \ell}: \bar{Z}_{5, i} \rightarrow \bar{Z}_{0, i}$ is a homeomorphism, so is $g^{2 \ell}: \bar{Y}_{5, i, n+2 \ell} \rightarrow \bar{Y}_{0, i, n}$, and if (2) holds,

$$
\bar{Y} \subset \bar{Y}_{5, i, n+2 \ell} \subset Y_{4, j, n+2 \ell} \subset g^{2 \ell} Y .
$$

This is enough to show that $Y$ is nonempty and homotopic to $P$ in $\overline{\mathbf{C}} \backslash X$. This is the set $Y$ that we need for the variation 2.3 on the Fixed Holed-Sphere Theorem, rather than the Fixed Holed-Sphere Theorem itself, for which we would need to replace $Y$ by a union of $\cap_{i=o}^{2 \ell} g^{i} Y$ and appropriate complementary components.

It remains to show (2). For this, it suffices to show that, for $n \geq N_{2}+i \ell k$, and $n \leq n^{\prime} \leq n+2 \ell k$,

$$
Y_{5, i+2, n^{\prime}} \subset Y_{5, i, n} \subset Y_{4, i, n} \subset Y_{4, i+2, n^{\prime}}, Y_{0, i+2, n^{\prime}} \subset Y_{0, i, n} .
$$


For then,

$$
\overline{\left\{\Psi_{n^{\prime}}(z): n^{\prime} \geq n\right\}} \subset \bar{Z}_{3} \subset Z_{1}
$$

whenever $z \in Y_{4, i, n}$, and so $\bar{Y} \subset Y_{1}$. Also,

$$
\bigcap_{n \geq N} \bigcap_{i} Y_{0, i, n}=\bigcap_{n \geq N_{2}} \bigcap_{i} Y_{0, i, n}
$$

if $N \geq N_{2}$. So $Y_{1} \subset \bigcap_{i} Y_{0, i, n}$ if $n \geq N_{2}$, and $\bar{Y}_{1} \subset Y_{0, i, n}$. To show (4), it suffices to find $N_{3}$ such that, for $n \geq N_{3}+i \ell k$, and any $x,\left\{\Psi_{n+t} x: 0 \leq t \leq 2 \ell k\right\}$ crosses at most one set of the form $G_{j}^{\prime}$ or $T^{2} G_{j}$ or $T^{2} G_{j}^{\prime}$, for $j \leq i+2$.

To complete the proof of the Fixed Holed-Sphere Theorem and its variations in this case, assuming (4) holds, we have to show that if $\gamma$ is any arc such that for all sufficiently large $n$, has $g^{n} \gamma$ arc length $\leq M$, and $\gamma \cap Y \neq \phi$, then $\gamma \subset Y$. It suffices to show this for $Y_{1}$ replacing $Y$, since $g^{2 \ell} Y$ is obtained by adding some discs to $Y_{1}$. So fix $x \in \gamma$, and suppose $\overline{\left\{\Psi_{n}(x): n \geq N\right\}} \subset Z_{1}$ for some $N$. We need to show there is $N^{\prime}$ such that $\Psi_{n}(\gamma) \subset Z_{1}$ for all $n \geq N^{\prime}$. But $\overline{\left\{\Psi_{n}(x): n \geq N\right\}} \subset Z_{2, i}$ for some $i$. So it suffices to find $N_{3} \geq N$ such that $\Psi_{n}(\gamma)$ crosses at most one set $T G_{s}$ for $s \leq j$ and $n \geq N_{3}+j \ell k$. For then $\Psi_{n}(\gamma) \subset Z_{2, i+2} \subset Z_{1}$ for $n \geq N_{3}+i \ell k$, and $\gamma \subset Y_{1}$, as required.

Now $\Psi_{n}(\gamma)$ and $\left\{\Psi_{n+t} x: 0 \leq t \leq \ell k\right\}$ are both components of the inverse image under $f^{n}$ of arcs of bounded length. So the proof of both (4) and the necessary fact about $\Psi_{n}(\gamma)$, and hence the proof of the theorem, is completed by the following lemma.

\subsection{Lemma.}

Given $M>0$, there is $N_{4}$ such that the following holds. If $\alpha$ is any arc of length $\leq M$, then, for $n \geq N_{4}$, any component of $f^{-n} \alpha$ intersects at most one component of $\overline{\mathbf{C}} \backslash \cup_{i \geq 0} f_{*}^{i} G_{0}$.

Proof: Recall that $\Gamma$ is a complete Levy set for $f$, and let $\Gamma_{1}=f^{-r} \Gamma$ for some suitable $r$, such that a nonperiodic loop of $\Gamma_{1}$ separates $Q_{1}$ and $Q_{2}$, whenever these are disjoint closed sets such that $Q_{i}$ is either a periodic loop in $\Gamma$ or the closure of a periodic gap for $\Gamma$ which is always mapped homeomorphically by $f^{n}$. This is clearly possible, because of the definition of complete Levy set (see Chapter 1) and because if $G_{0}$ is periodic, only one boundary component is periodic. Then it is clear that there exists $L$ for which the following is true. For any $\alpha$ of length $\leq M$, there exist $L^{\prime}=L^{\prime}(\alpha) \leq L$ and $\alpha_{i}\left(0 \leq i \leq L^{\prime}\right)$ with $\alpha=\cup_{i=1}^{L^{\prime}} \alpha_{i}$, such that the arc $\alpha_{i}$ is homotopic in $\overline{\mathbf{C}} \backslash X$, via a homotopy fixing endpoints, to one with at most one intersection with a loop in $\Gamma_{1}$. Then there is $N_{4}$ (independent of $\alpha$ ) such that for $n \geq N_{4}$, any component of $g^{-n} \alpha$ intersects at most one element of $\Gamma_{1}$, since it intersects at most $L^{\prime}$ elements of $g^{-n} \Gamma_{1}$. Then each one can intersect at most one component of $\overline{\mathbf{C}} \backslash \cup_{i \geq 0} f_{*}^{i} G_{0}$, since the boundary of each $f_{*}^{i} G_{0}$ is contained in $\Gamma \subset \Gamma_{1}$. 


\subsection{Proof of the Fixed Holed-Sphere Theorem: a simpler analogue of the Nonrational Adjacent Case.}

Let $g: \mathbf{R}^{2} / \mathbf{Z}^{2} \rightarrow \mathbf{R}^{2} / \mathbf{Z}^{2}$ be a covering of the two-torus homotopic to an endomorphism given by a matrix $A$ in $G L(2, \mathbf{Z})$, where $A$ has determinant 2 and eigenvalues $\lambda_{u}, \lambda_{s}$ with $\left|\lambda_{s}\right|<1<2<\left|\lambda_{u}\right|$. Let $v_{u}$, $v_{s}$ be the corresponding eigenvectors. Let $\bar{g}$ denote a (well-defined) lift of the multivalued function $g^{-1}$ to $\mathbf{R}^{2}$ (so that notation agrees with what we shall do later). Then let

$$
\begin{aligned}
& V=\left\{x v_{u}+y v_{s}:|x|<M^{\prime}\right\}, \\
& W=\left\{x v_{u}+y v_{s}:|y|<M^{\prime}\right\},
\end{aligned}
$$

for some suitable $M^{\prime}>0$. We have $A W \subset W$, because $A\left(x v_{u}+y v_{s}\right)=$ $x \lambda_{u} v_{u}+y \lambda_{s} v_{s}$, and similarly $A^{-1} V \subset V$. In fact, the distance between the boundaries of $W$ and $A W$ increases with $M^{\prime}$, and similarly with $V, A^{-1} V$. But there is $M$ such that distance $\left(\bar{g} x, A^{-1} x\right) \leq M$ for all $x$. So if $M^{\prime}$ is sufficiently large given $M$, we can ensure that $\bar{g} V \subset V$, and similarly $W \subset \bar{g} W$.

Now we consider the set $\bar{g}^{n}(V \cap W)=Y_{n}$, so that $Y_{n+1}=\bar{g} Y_{n}$. Let

$$
B_{n}=\left\{z \in \mathbf{Z}^{2}: x, x+z \in Y_{n} \cup Y_{n+1} \text { for some } x\right\} .
$$

Then $B_{n}=A^{-n} B_{0} \cap \mathbf{Z}^{2}$. We claim that $\cap_{n=0}^{\infty} A^{n} \mathbf{Z}^{2}=\{0\}$. For $A$ is an automorphism of the subgroup $\cap_{n=0}^{\infty} A^{n} \mathbf{Z}^{2}$, which must then be either $\{(0,0)\}$ or rank two, since no nontrivial rationally defined subspace is invariant under $A$. But $A$ has determinant 2 , so this subgroup must indeed be $\{(0,0)\}$. (I would like to thank my colleagues for helping me clarify this rather trivial point.) So, since $B_{0}$ is finite, $B_{n}=\{(0,0)\}$ for all sufficiently large $n$. So for sufficiently large $n, Y_{n} \cup Y_{n+1}$ projects homeomorphically to $\mathbf{R}^{2} / \mathbf{Z}^{2}$, and $g$ maps the projection of $Y_{n+1}$ homeomorphically to the projection of $Y_{n}$. Moreover, if $Y$ denotes the projection of $Y_{n+1}$, we can write $\partial Y=\partial_{1} \cup \partial_{2}$, where each $\partial_{j}$ is the union of two disjoint arcs, and $g \partial_{1} \cap Y=\phi, g Y \cap \partial_{2}=\phi$. This is about as far as we can take the arguments of $[\mathbf{F r}]$. We now want to generalise this argument, to prove the Nonrational Adjacent case of the Fixed Holed-Sphere Theorem.

\subsection{Proof of the Fixed Holed-Sphere Theorem in the Nonrational Ad- jacent Case: lifting the branched covering inverse to the unit disc.}

We are working under the following assumptions. The map $g: \overline{\mathbf{C}} \rightarrow \overline{\mathbf{C}}$ is a degree two critically finite orientation-preserving branched covering, with nonrational critical branched covering and an adjacent periodic set. (See the Decomposition Proposition.) Thus, there is a homotopy $\left\{f_{t}: t \in[0,1]\right\}$ through critically finite branched coverings with $f_{0}=g, f_{1}=f, X\left(f_{t}\right)=X(f)=X$ for all $t \in[0,1]$, and $f$ has the following properties.

The set $Q$. There is a set $Q$ such that $f^{m} Q=Q$, for some $m \geq 1$. Either $Q$ is a loop, in which case $f^{m}$ reverses orientation on $Q$ and we write $\ell=2$, or $Q$ 
is a $\ell$-holed sphere (for some $\ell \geq 3$ ). In the latter case, $f^{m}$ cyclically permutes the components of $\partial Q$, and $f^{q}$ fixes the boundary components, where $q=m \ell$.

The sets $G, \widehat{G}$. Either way, there is a set $G$ adjacent to $Q$ with the following properties. The sets $f^{i} G(0 \leq i \leq q)$ are disjoint for $i<q$, and adjacent to $Q$ when $m \mid i$, with $G \subset f^{q} G, \partial f^{q} G \subset \partial G$. Also, $G$ has a holed torus as a double covering. There is a holed plane $\widehat{G}$ covering this torus, and $f^{-q} \mid G$ lifts to $\widehat{f}: \widehat{G} \rightarrow \widehat{G}$ having the following properties. The map $\widehat{f}$ is injective, but not surjective, and there are a $C^{1} \varphi: \widehat{G} \rightarrow \mathbf{R}^{2}$ and $A \in G L(2, \mathbf{Z})$, with determinant 2 , such that

$$
\varphi=A \circ \varphi \circ A c c \hat{f} .
$$

Moreover, $\varphi$ conjugates the action of the covering group of the torus to the action of $\mathbf{Z}^{2}$ on $\mathbf{R}^{2}$ by translation. The eigenvalues $\lambda_{u}, \lambda_{s}$ of $A$ satisfy $\left|\lambda_{s}\right|<1<$ $2<\left|\lambda_{u}\right|$. We fix corresponding eigenvectors $v_{u}, v_{s}$.

Lifting Inverses, and Paths Representing Points in the Universal Cover. Now we recall how to lift $f_{t}^{-n}(n \geq 1)$ to the universal cover $\stackrel{\circ}{D}$ of $\overline{\mathbf{C}} \backslash X$ (This lifting of the inverses of critically finite branched coverings is fundamental in [T].) We fix a basepoint $x_{1}$ in $\overline{\mathbf{C}} \backslash X$ - which, for convenience, we take in $Q$. Fix $n$, and fix $x_{1}^{\prime}$ with $f_{1}^{n} x_{1}^{\prime}=x_{1}$, hence fixing a path $\left\{x_{t}^{\prime}\right\}$ with $f_{t}^{n} x_{t}^{\prime}=x_{1}$. We also fix a path $\alpha_{1}$ from $x_{1}$ to $x_{1}^{\prime}$. Then $\alpha_{t}$ is the path $\alpha_{1}$ followed by $\left\{x_{u}^{\prime}\right\}$ from $x_{1}^{\prime}$ to $x_{t}^{\prime}$. Then $\stackrel{\circ}{D}$ can be identified with homotopy classes of paths in $\overline{\mathbf{C}} \backslash X$ based at $x_{1}$. If $\beta$ is any such path, let $\beta_{t}$ be the component of $f_{t}^{-n} \beta$ based at $x_{t}^{\prime}$. We write

$$
\bar{f}_{t}[\beta]=\left[\alpha_{t} * \beta_{t}\right]
$$

where $[\beta]$ is the homotopy class of $\beta$. Then $\bar{f}_{t}: \stackrel{\circ}{D} \rightarrow \stackrel{\circ}{D}$ is well-defined.

The group $\Delta$. If $\bar{f}_{t}[\beta]=\bar{f}_{t}[\gamma]$, and $[\beta] \neq[\gamma]$ then $[\beta]=[\tau * \gamma]=[\tau] \cdot[\gamma]$, where $\tau$ is a closed loop in $\overline{\mathbf{C}} \backslash X$ based at $x_{1}$ (so that $[\tau] \in \pi_{1}(\overline{\mathbf{C}} \backslash X)$ ). The converse is not necessarily true. The set of $\tau$ which arises is independent of $t$. Similarly, if $\bar{f}_{t}[\beta]=[\tau] . \bar{f}_{t}[\gamma]$ for some $\tau \in \pi_{1}(\overline{\mathbf{C}} \backslash X)$, then $[\beta]=[\sigma] \cdot[\gamma]$, where $[\sigma] \in \pi_{1}(\overline{\mathbf{C}} \backslash X)$ is again independent of $t$. Let $\Delta$ be the subgroup of $\pi_{1}(\overline{\mathbf{C}} \backslash X)$ consisting of all $[\delta]$ such that $\bar{f}^{n}([\delta] . x) \in \pi_{1}(\overline{\mathbf{C}} \backslash X) \cdot \bar{f}^{n}(x)$ for all $x$, for all $n$. Then $\pi_{1}(Q)$, $\pi_{1}(\widehat{G}) \leq \Delta$. From the definition of $\bar{f}_{t}[\beta]$, if $\bar{f}_{t}[\beta]=[\gamma]$, there is $\delta$ with initial point at $x_{t}^{\prime}$, and

$$
\begin{gathered}
{\left[\alpha_{t}^{\prime} \cup \delta\right]=[\gamma],} \\
{\left[f_{t}^{n} \delta\right]=[\beta] .}
\end{gathered}
$$




\subsection{The construction of $V_{t}$ and $W_{t}$.}

From now on, we take $\bar{f}_{t}$ to be a lift of $f_{t}^{-m}$, with $\bar{f}=\bar{f}_{1}$ and $\bar{g}=\bar{f}_{0}$. Let $\widetilde{Q}$ be a fixed lift of $Q$, and let $\widetilde{G}$ be a lift of $G$ adjacent to $\widetilde{Q}$. By taking $x_{1}$, $x_{1}^{\prime} \in Q$ and $\alpha_{1} \subset Q$, we can ensure that $\bar{f} \widetilde{Q}=\widetilde{Q}$.

Now we are ready to construct $\partial V_{t}$ by constructing the $\Delta$-orbit $\partial_{t}$ of its boundary, and then defining $V_{t}$ to be the component of $\stackrel{\circ}{D} \backslash \partial_{t}$ containing $\widetilde{Q}$. We define $\partial_{t}=\cup_{i=0}^{\ell-1} \bar{f}_{t}^{i} \partial^{\prime}$ where $\partial^{\prime}$ is $\Delta$-invariant, and is defined by its intersection with $\widetilde{G}$. Since $\pi_{1}(\widehat{G}) \leq \Delta$, it suffices to construct the projections of the components to $\widehat{G}$. To do this, it suffices to define the images under $\varphi$ in $\mathbf{R}^{2}$, provided these are disjoint from $\varphi(\partial \widehat{G})$.

We take the images under $\varphi$ of projections of components of $\partial^{\prime}$ to $\widehat{G}$ to be

$$
\left\{x v_{u}+y v_{s}: x= \pm M^{\prime}\right\} .
$$

The definition of $W_{t}$ is similar, with $v_{u}$ and $v_{s}$ interchanged.

\subsection{Lemma.}

$\bar{f} V_{1} \subset V_{1}$ and given $M>0, M^{\prime}$ can be chosen so that $d_{P}\left(\partial V_{1}, \bar{f} \partial V_{1}\right)>M$, where the $d_{P}$ is the Poincaré metric. Similarly, $W_{1}$ contains a component of $\bar{f}^{-1} W_{1}$ containing $\widetilde{Q}$, and $d_{P}\left(\partial W_{1}, \bar{f} \partial W_{1}\right)>M$.

Proof: We represent points in $\stackrel{\circ}{D}$ by homotopy classes of paths in $\overline{\mathbf{C}} \backslash X$ with first endpoints at $x_{0} \in Q$. We shall use the same letters to represent the lifts of the paths to the universal cover $\stackrel{\circ}{D}$. We consider the case of $\partial V_{1}$ - the proof for $\partial W_{1}$ is exactly similar. So first, let $\alpha$ be a path representing an element of $V_{1}$, with first endpoint in $Q$. Thus, $\alpha \subset V_{1}$. We have to show $\bar{f} \alpha \subset V_{1}$. By Lemma 2.14 below, for all sufficiently large $n, \bar{f}^{n} \alpha$ has only one component of intersection with $Q$ and none with any other component of $\overline{\mathbf{C}} \backslash \cup_{i=0}^{\ell-1} f^{i m} G$ (assuming that $\alpha$ has only essential intersections with components of $\overline{\mathbf{C}} \backslash \cup_{i=0}^{\ell-1} f^{i m} G$ ). For $n$ divisible by $\ell$ (without loss of generality), $\bar{f}^{n} \alpha \subset Q \cup G$, and $\bar{f}^{n} \alpha$ does not cross $\bar{f}^{n} \partial_{1}$, because $\partial_{1}=\bar{f}^{-n}\left(\bar{f}^{n} \partial_{1}\right)$. In $\widetilde{G}$ itself, $\bar{f}^{n} \partial_{1}$ separates $\bar{f}^{n-1} \partial_{1}$ from $\widetilde{Q}$. So $\bar{f}^{n} \alpha$ also does not cross $\bar{f}^{n-1} \partial_{1}$, and so $\bar{f} \alpha$ does not cross $\partial_{1}$. Hence $\bar{f} \alpha \subset V_{1}$, as required.

To show $d_{P}\left(\partial V_{1}, \bar{f} V_{1}\right)>M$, suppose not, and let $\alpha$ be a path of length $\leq M$ between the boundary components. Again by 2.14, for some $n$ and $M_{1}$ depending only on $M, \bar{f}^{n} \alpha$ is a path of length $\leq M_{1}$ which may have one component of intersection with $Q$, but intersects no other component of $\overline{\mathbf{C}} \backslash$ $\cup_{i=0}^{\ell-1} f^{i m} G$ (again, assuming only essential intersections for $\alpha$ ). Suppose without loss of generality that part of $\bar{f}^{n} \alpha$ with endpoint lifting to $\bar{f}^{n} \partial V_{1}$ lies in $G$, and hence lifts to $\widehat{G}$, with endpoint in $\varphi\left(\widehat{f}^{n} \partial^{\prime}\right)$. But because $\Delta \cap \pi_{1}(G)=\pi_{1}(\widehat{G}), \bar{f}^{n} \alpha$ cannot intersect $Q$ in just one component. So we can choose $n$ so that $\bar{f}^{n} \alpha \subset G$, 
and then obtain a bound (in terms of $M$ ) on the distance between components of $\bar{f}^{n} \partial_{1} \cap \widetilde{G}$ and $\bar{f}^{n+1} \partial_{1} \cap \widetilde{G}$, which can be contradicted by choice of $M^{\prime}$.

2.14. Lemma. Let $M$ be given. Then there is $r$ such that, if $\gamma:[0,1] \rightarrow \overline{\mathbf{C}} \backslash X$ is any path of length $\leq M$, the following holds after homotopy of $\gamma$ in $\overline{\mathbf{C}} \backslash X$ fixing the endpoints. For $n \geq r$, a lift of $\gamma$ under $f^{n}$ has at most one component of intersection with $Q$, and none with any other component of $\overline{\mathbf{C}} \backslash \cup_{i=0}^{\ell-1} f^{i m} G$.

Proof: We can homotope $\gamma$, fixing endpoints, to have $m_{0}<\infty$ intersections with $\cup_{i=0}^{\ell-1} f^{i m} \partial G$, all essential in $\overline{\mathbf{C}} \backslash X$. Let $m_{n}$ be the maximal number of intersections for a lift of $\gamma$ under $f^{n}$. Then since the intersections for a lift under $f^{n}$ are inverse images under $f^{n}$ of the intersections for $\gamma,\left\{m_{n}\right\}$ is a decreasing sequence, and there is $r_{1}$ such that, for $n \geq r_{1}$, the intersections are only with components of $\partial Q$. Now if $\gamma^{\prime}$ is any path of length $\leq M_{2}$ with endpoints in $\partial Q$ but otherwise lying in some $f^{i m} G$, there is $r_{2}$ (depending only on $M_{2}$ ) such that, for $n \geq r_{2}$, no lift of $\gamma^{\prime}$ under $f^{n}$ has both endpoints in $\partial Q$. Applying this repeatedly, we obtain the required $r$.

\subsection{Properties of $V_{t}, W_{t}$.}

Note that there is $M$ such that, for $0 \leq i \leq \ell$,

$$
d_{P}\left(\bar{f}^{i} x, \bar{f}_{t}^{i} x\right)<M
$$

Then if $M^{\prime}$ is sufficiently large given $M$, we have the following corollary of 2.13.

\section{Corollary.}

$\bar{f}_{t}^{n+1} \bar{V}_{t} \subset \bar{f}_{t}^{n} V_{t}, \bar{f}_{t}^{n} \bar{W}_{t} \subset \bar{f}_{t}^{n+1} W_{t}, \partial \bar{f}_{t}^{n} V_{t}=\bar{f}_{t}^{n} \partial V_{t}$, and $\partial \bar{f}_{t}^{n} W_{t}=\bar{f}_{t}^{n} \partial W$ for all $t \in[0,1]$ and $n \geq 0$.

Proof: By 2.13 and (1) above, $\bar{f}_{t} \partial V_{t} \subset \bar{V}_{t}$. The last two statements follow from the fact that $\partial\left(\Delta \cdot \bar{f}_{t}^{n} V_{t}\right)=\Delta \cdot \bar{f}_{t}^{n} \partial V_{t}$, and similarly for $W_{t}$. This is easily proved by induction: the point is that $\bar{f}_{t}$ is a covering from $\Delta \cdot \bar{f}_{t}^{n} \bar{V}_{t}$ onto $\Delta \cdot \bar{f}_{t}^{n+1} \bar{V}_{t}$, and

similarly for $W_{t}$. Hence $\bar{f}_{t} V_{t} \subset V_{t}$ and by induction $\bar{f}_{t}^{n+1} V_{t} \subset \bar{f}_{t}^{n} V$. The proof that $\bar{f}_{t}^{n} W \subset \bar{f}_{t}^{n+1} W_{t}$ is similar, and actually slightly easier.

\subsection{Proof of the Fixed Holed-Sphere Theorem in the Nonrational Adjacent Case.}

Note that $V_{1} \cap W_{1}$ has the following property: for some integer $N, \bar{f}^{n}\left(\bar{V}_{1} \cap\right.$ $\bar{W}_{1}$ ) projects to a compact subset of $\stackrel{\circ}{D} / \pi_{1}(Q)$ for $n \geq N$. This is simply because, for some $N$ the set

$$
A^{-n}\left(\left\{x v_{u}+y v_{s}:|x| \leq M^{\prime},|y| \leq M^{\prime}\right\}\right)
$$


does not intersect $\mathbf{Z}^{2} \backslash\{(0,0)\}$ for $n \geq N$. We claim that $\bar{f}_{t}^{n}\left(\bar{V}_{t} \cap \bar{W}_{t}\right)$ also projects to a compact subset for all $t$. For

$$
\bar{f}_{t}^{n}\left(\bar{V}_{t} \cap \bar{W}_{t}\right) \subset \bar{f}^{n}\left(\bar{V}_{1} \cap \bar{W}_{1}\right) \cup \bigcup_{0 \leq i<\ell, t \leq u \leq 1} \bar{f}_{u}^{i}\left(\psi_{u} \bar{f}^{n} \partial\left(V_{1} \cap W_{1}\right)\right) .
$$

Here, $\psi_{u}$ is a lift of the homeomorphism $f_{u}^{-n} \circ f^{n}$ to $\stackrel{\circ}{D} / \pi_{1}(Q)$. So the claim is proved. So, in particular, $\bar{g}^{N}\left(V_{0} \cap W_{0}\right)=[\tau] \cdot \bar{g}^{N}\left(V_{0} \cap W_{0}\right)$ for $[\tau]$ lying in only finitely many cosets of $\pi_{1}(Q)$. Then 2.14 implies that by enlarging $N$, we can assume this is true only for $[\tau] \in \pi_{1}(Q)$. Take $Y$ to be the projection of $\bar{g}^{N}\left(V_{0} \cap W_{0}\right)$ to $\overline{\mathbf{C}} \backslash X$. Let $\partial_{1}$ be the projection of $\bar{g}^{N}\left(\partial V_{0} \cap \partial\left(V_{0} \cap W_{0}\right)\right)$, and $\partial_{2}$ the projection of $\bar{g}^{N}\left(\partial W_{0} \cap \partial\left(V_{0} \cap W_{0}\right)\right)$. If we isotope $\partial^{\prime}$ (see 2.12) a distance $\leq M$ in a $\Delta$-invariant way, and redefine $V_{t}$ using the new definition of $\partial^{\prime}$, then $V_{t}$ still has the properties claimed above, and similarly for $W_{t}$. So we can assume that each component of $\partial V_{0}$, or $\bar{g} \partial V_{0}$ intersects each component of $\partial W_{0}$ at most once, by choice of $\partial^{\prime}$ and the corresponding set for $\partial W_{0}$. Then $Y$ has all the properties stated in the Fixed Holed-Sphere Theorem.

\subsection{Proof of the Variations in the Nonrational Adjacent Case.}

For any $p>0$, the set $\cup_{i=0}^{p} \bar{g}^{N-i}\left(V_{0} \cap W_{0}\right)$ intersects its image under $[\tau]$ only for $[\tau] \in \pi_{1}(Q)$ if $N$ is large enough. We can also make the distance in $\bar{g} W_{0}$ between $\partial W_{0}$ and $\bar{g} \partial W_{0}$ arbitrarily large by choice of $M^{\prime}$ - and then the distance in $\bar{g}^{n+1} W_{0}$ between $\bar{g}^{n} W_{0}$ and $\bar{g}^{n+1} \partial W_{0}$ is even larger, for any $n>0$. If $g$ is a lamination map, we can choose $\partial^{\prime}$ (and the analogue used to define $\partial W_{0}$ ) to be a union of lifts of $S^{1}$-segments and leaf segments, and by tracing round the boundaries of gaps, can avoid the lifts of the forward orbits of the $S^{1}$-segments $I_{i}$ (see variation 3 of 2.3). Then $Y$ has all the required properties. 


\section{Chapter 3. One-dimensional Topological Dynamics.}

\subsection{Definitions.}

Let $T$ be a locally finite graph. If $x \in T, U \subset T$ is a 1 -sided neighbourhood of $x$ if $U \backslash\{x\}$ is connected, nonempty, contains no vertex of $T$, and $x \in U$. (To fit in with what we need to prove, this definition and the following one may be a bit unorthodox.) Let $h: T \rightarrow T$ be continuous with $h x=x$. Then we define $W^{u}(x, h, U)$ by

$$
W^{u}(x, h, U)=\{x\},
$$

if arbitrarily small 1 -sided neighbourhoods $V$ of $x$ in $U$ satisfy $h V \backslash U \neq \phi$, and

$$
W^{u}(x, h, U)=\bigcap\left\{\bigcup_{n \geq 0} h^{n} V: V \text { is a } 1 \text {-sided neighbouhood of } x, V \subset U\right\}
$$

otherwise. Thus $W^{u}\left(x, h, U_{1}\right)=W^{u}\left(x, h, U_{2}\right)$ if $U_{1}, U_{2}$ are 1 -sided neighbourhoods with $U_{1} \cap U_{2} \neq \phi$.

\section{2 .}

The main purpose of this chapter is to prove the following.

\section{The 1-sided Neighbourhood Theorem.}

Let $Y \subset \overline{\mathbf{C}}$ be an $\ell$-holed sphere with boundary $(\ell \geq 2)$. Let $T \subset Y$ be a graph such that $T \hookrightarrow Y$ is a homotopy equivalence. Let $\chi: Y \rightarrow Y$ be an orientation-preserving homeomorphism which cyclically permutes the components of $\partial Y$. Let $h: T \rightarrow T$ be continuous and homotopic to $\chi \mid T$ as a map from $T$ to $Y$.

Then there is a graph $T_{1} \subset T$ with no free vertices such that $\overline{\mathbf{C}} \backslash T_{1}$ has $k$ components (for some $2 \leq k \leq \ell$ ), $h \mid T_{1}$ is homotopic as a map from $T_{1}$ to $T$ to some $h_{0}: T_{1} \rightarrow T_{1}$ which cyclically permutes the boundaries of the components of $\overline{\mathbf{C}} \backslash T_{1}$ up to homotopy, and

$$
\begin{gathered}
T_{1} \subset \bigcup\left\{W^{u}\left(x, h^{k}, U\right): h^{k} x=x, x \in T_{1}, U \text { is a } 1\right. \text {-sided } \\
\text { neighbourhood of } \left.x \text { in } T_{1}\right\}
\end{gathered}
$$

\section{3 .}

The main tool in the proof of the 1-sided Neighbourhood Theorem is the Pseudo-Anosov Proposition below. Some of the same ideas occurred in the proof of the Tuning Proposition in Chapter 7 of [R2], and, as there, we rely heavily on Thurston's description of surface homeomorphisms up to isotopy, as described in [F-L-P]. 


\section{Pseudo-Anosov Proposition.}

Let $Y_{1}$ be a $k$-holed sphere $(k \geq 2)$. Let $\chi: Y_{1} \rightarrow Y_{1}$ be a homeomorphism which cyclically permutes the boundary components of $Y_{1}$ and does not leave isotopically invariant any nontrivial nonboundary disjoint loop set in $Y_{1}$. Suppose also that $\chi$ fixes a point $x_{0}$ in $Y_{1}$. Then $\chi$ is isotopic, via an isotopy fixing $x_{0}$, to $\chi_{0}: Y_{1} \rightarrow Y_{1}$ satisfying one of the following.

1. $\chi_{0}^{k}=$ identity.

2. $\chi_{0}$ fixes a disc $D$ round $x_{0}$, and $\chi_{0}: Y_{2} \rightarrow Y_{2}$ is pseudo-Anosov, where $Y_{2}=$ $Y_{1} \backslash D$. In particular, a measured foliation on $Y_{2}$ with singularities is preserved by $\chi_{0}$, and this foliation has singular leaf $\ell$ with endpoint at $x_{1} \in \partial Y_{1} \subset \partial Y_{2}$ such that $x_{1}, \ell$ are preserved by $\chi_{0}^{k}$. Moreover, there is a length measurement defined on $\ell$, and $\lambda>1$, such that $\chi_{0}^{k}$ multiplies length on $\ell$ by $\lambda$, and the length of any segment of $\ell$ is proportional to the diameter of a lift to $\widetilde{Y}_{2}$, where $\widetilde{Y}_{2}$ is the universal cover of $Y_{2}$.

Proof: To follow the outline of [F-L-P] more closely, we assume (as we may do) that $\chi$ preserves $D$. The Teichmuller space of $Y_{2}=Y_{1} \backslash D$ is then the space of (non-complete) hyperbolic structures on $Y_{2}$ for which all the boundary components are geodesics of length 1 . This space is homeomorphic to the open unit ball in $\mathbf{R}^{2 k-4}$ (or a single point if $k=2$ ) and its Thurston compactification is homeomorphic to the closed unit ball in $\mathbf{R}^{2 k-4}$. Exactly as in $[\mathbf{F}-\mathbf{L}-\mathbf{P}], g$ induces a homeomorphism of this Thurston compactification (the only difference is that, here, $\chi$ does not fix boundary components) and must have a fixed point. Since no simple disjoint nontrivial nonboundary loop set in $Y_{1}$ is left invariant by $\chi$ up to isotopy, and $\chi$ cyclically permutes the components of $\partial Y_{1}$, there is similarly no such loop set in $Y_{2}$. So there is $\chi_{0}$ isotopic to $\chi$ such that either

1. $\chi_{0}$ is an isometry, for some hyperbolic structure on $Y_{2}$, or

2. $\chi_{0}$ is pseudo-Anosov, in a sense made more precise below (and see [F-L-P]). Case 1. We claim that $\chi_{0}^{k}=$ identity on $Y_{2}$, and we can then extend $\chi_{0}$ to $Y_{1}$ with $\chi_{0}^{k}=$ identity on $Y_{1}$. For let $\gamma$ be a geodesic arc of minimal length joining $\partial D$ to $\partial Y_{1}$. Then $\gamma$ is disjoint from all other such arcs. It follows that $\chi_{0}^{i} \gamma$ $(0 \leq i<k)$ are disjoint, and $\chi_{0}^{k} \gamma=\gamma$. Since $Y_{1} \backslash\left(D \cup \bigcup_{i \geq 0} \chi_{0}^{i} \gamma\right)$ is a disc, $\chi_{0}^{k}=$ identity on $Y_{2}$.

Case 2. We are only really interested in the unstable foliation preserved by $\chi_{0}$. This singular foliation has finitely many singularities, at least one on each component of $\partial Y_{2}$, and the complement of the singularities in $\partial Y_{2}$ is a finite set of leaves. The set of singularities is also preserved by $\chi_{0}$. There is a length measurement on leaves in Interior $\left(Y_{2}\right)$ (induced by the unstable foliation being transverse to the stable), and there is $\lambda>1$ such that $\chi_{0}$ multiplies length by $\lambda$. Let $x_{i}(1 \leq i \leq r)$ be the singularities. There is an integer $p_{i} \geq 3$ and a chart

$$
\{z:|z|<1\} \text { if } x_{i} \in \operatorname{Interior}\left(Y_{2}\right)
$$




$$
\{z:|z|<1, \operatorname{Re}(z) \geq 0\} \text { if } x_{i} \in \partial Y_{2},
$$

with $x_{i}$ corresponding to 0 in the chart, such that the leaves of the foliation are locally the level sets of the multivalued function

$$
\begin{gathered}
\operatorname{Re}\left(z^{p_{i} / 2}\right) \text { if } x_{i} \in \operatorname{Interior}\left(Y_{2}\right), \\
\operatorname{Re}\left(z^{p_{i}-1}\right) \text { if } x_{i} \in \partial Y_{2} .
\end{gathered}
$$

Then by the Poincaré-Hopf Theorem,

$$
\sum_{i=1}^{r}\left(\frac{p_{i}}{2}-1\right)=k-1
$$

Since the number of singularities on each component of $\partial Y_{1}$ is the same (because $\chi_{0}$ preserves the set of singularities) it follows from (1) that there must be exactly one singularity on each component of $\partial Y_{1}$, and these are of the form $\chi_{0}^{i} x_{1}(0 \leq$ $i<k$ ) for some $x_{1} \in \partial Y_{1}$ with $\chi_{0}^{k} x_{1}=x_{1}$, and the $p_{i}$ associated to $x_{1}$ must be 3 , so that exactly one leaf $\ell$ in Interior $\left(Y_{2}\right)$ ends at $x_{1}$, and $\chi_{0}^{k} \ell=\ell$. Also, from (1) and the preservation of the foliation by $\chi_{0}$, we deduce that $\ell$ meets no other singularities. Also, since all infinite half-leaves of the unstable foliation in Interior $\left(Y_{2}\right)$ are dense, $\bar{\ell}=Y_{2}$. Since any measured foliation is recurrent, there is $M_{0}$ such that every segment of $\ell$ of length $M_{0}$ meets an element of $\Gamma$, where $\Gamma$ is a fixed collection of disjoint simple closed curves on $Y_{2}$ cutting $Y_{2}$ into pairs of pants. It follows that the length of any segment $\ell_{1}$ of $\ell$ is proportional to the diameter of any lift of it to $\widetilde{Y}_{2}$.

\subsection{Proof of the 1-sided Neighbourhood Theorem: finding $Y_{1}, T_{1}$, $x_{0}, \chi_{0}$.}

Since, by the Lefschetz Theorem, $\chi$ has a fixed point in $Y$, we can choose a connected subsurface $Y_{1}$ of $Y$ which is invariant under $\chi$ up to isotopy, and such that no nontrivial nonboundary simple disjoint loop set in $Y_{1}$ is preserved by $\chi$ up to isotopy. Then we can assume $\chi Y_{1}=Y_{1}$. Then $\chi$ cyclically permutes the components of $\partial Y_{1}$, since $\chi$ cyclically permutes the components of $Y \backslash Y_{1}$. We can also assume there is a subgraph $T_{1} \subset T$ with no free vertices such that $T_{1} \subset Y_{1}$ and $T_{1} \hookrightarrow Y_{1}$ is a homotopy equivalence. So there is $p: Y_{1} \rightarrow T_{1}$ such that $p \mid T_{1}$ is homotopic to the identity. Let $\widetilde{Y}, \widetilde{T}, \widetilde{Y}_{1}, \widetilde{T}_{1}$ be the universal covers of $Y, T, Y_{1}, T_{1}$ with $\widetilde{T}_{1} \subset \widetilde{Y}_{1} \subset \widetilde{Y}$ and $\widetilde{T}_{1} \subset \widetilde{T} \subset \widetilde{Y}$. Let $d$ be a fixed metric on $\widetilde{Y}$ (lifted from a metric on $Y$ ) invariant under the action of the fundamental group $\pi_{1}(Y)$. Let $\widetilde{h}, \widetilde{\chi}, \widetilde{p}$ be the lifts of $h, \chi, p$ so that a homotopy between $p \circ \chi$ and $h$ (as maps from $T_{1}$ to $T$ ) lifts to a homotopy between $\widetilde{h}$ and $\widetilde{p} \circ \widetilde{\chi}$. Then there is $M_{1}>0$ so that

$$
d(\widetilde{h}(x), \widetilde{p} \circ \widetilde{\chi}(x)) \leq M_{1} \text { for all } x \in \widetilde{T} .
$$


Since $\widetilde{h}\left(\widetilde{T}_{1}\right)$ is connected, for any $x_{1}, x_{2} \in \widetilde{T}_{1}, \widetilde{h}\left(\widetilde{T}_{1}\right)$ contains the unique arc between $\widetilde{h} x_{1}$ and $\widetilde{h} x_{2}$. Then we claim that

$$
\widetilde{T}_{1} \subset \widetilde{h} \widetilde{T}_{1} .
$$

For given $y \in \widetilde{T}_{1}$, we can find $x_{1}, x_{2} \in \widetilde{T}_{1}$ such that $\widetilde{p} \circ \widetilde{\chi}\left(x_{1}\right), \widetilde{p} \circ \widetilde{\chi}\left(x_{2}\right)$ are separated by $y$ and both distance $>M_{1}$ from $y$. Then $y$ also separates $\widetilde{h} x_{1}$ and $\widetilde{h} x_{2}$. So $y \in \widetilde{h} T_{1}$. Then a continuous map $\widetilde{h}_{0}: \widetilde{T}_{1} \rightarrow \widetilde{T}_{1}$ is uniquely determined by the properties

$$
\widetilde{h}_{0}=\widetilde{h} \text { on } \widetilde{T}_{1} \cap \widetilde{h}^{-1} \widetilde{T}_{1},
$$

$\widetilde{h}_{0}$ maps each component of $\widetilde{T}_{1} \backslash \widetilde{h}^{-1} \widetilde{T}_{1}$ to a point.

Then $\widetilde{h}_{0}$ descends to a map $h_{0}: T_{1} \rightarrow T_{1}$ which is homotopic to $\chi: T_{1} \rightarrow Y_{1}$. So by the Lefschetz Fixed Point Theorem, $h_{0}$ has a fixed point $x_{0}$. Then we can assume that $\chi\left(x_{0}\right)=x_{0}$, and that $\chi \mid T_{1}$ and $h_{0}: T_{1} \rightarrow Y_{1}$ are homotopic via a homotopy fixing $x_{0}$. Then we can find $\chi_{0}: Y_{1} \rightarrow Y_{1}$ as in the Pseudo-Anosov Proposition.

\subsection{Proof of the 1-sided Neighbourhood Theorem: a reformulation.}

It suffices to prove, for some lift $H$ of $h^{k}$ to $\widetilde{T}$,

$$
\widetilde{T}_{1} \subset \bigcup\left\{\tau . W^{u}(x, H, U): x \in \widetilde{T}_{1}, H x=x, U \text { is a } 1\right. \text {-sided }
$$

neighbourhood of $x$ in $\widetilde{T}_{1}$, and $\left.\tau \in \pi_{1}\left(T_{1}\right)\right\}$.

For, projecting down, this proves the theorem.

\subsection{Lemma.}

\section{It suffices to prove:}

(3.6) for some closed arc $I$ in $\widetilde{T}_{1}$ projecting down to cover $T_{1}$ and some lift $H^{\prime}$ of $h_{0}^{k}, I \subset \bigcup\left\{W^{u}\left(x, H^{\prime}, U\right): x \in I, H^{\prime} x=x\right.$ and $U$ is a 1 -sided neighbourhood of $x$ in $I\}$.

Proof: Suppose (3.6) holds. Then let $\tau \in \pi_{1}\left(T_{1}\right)$ be such that $H^{\prime}=\tau \cdot \widetilde{h}_{0}^{k}$, and let $H=\tau . \widetilde{h}^{k}$. We claim that a), b) and c) hold. Clearly they suffice to prove (3.5), as is required.

a) $W^{u}\left(x, H^{\prime}, U\right) \subset W^{u}(x, H, U)$ if $H^{\prime} x=x$ and $\widetilde{h}_{0}^{i} x=\widetilde{h}^{i} x$ for $1 \leq i \leq k$,

b) $W^{u}\left(x, H^{\prime}, U\right)=\{x\}$ if $H^{\prime} x=x$ and $\widetilde{h}_{0}^{i} x \neq \widetilde{h}^{i} x$ for some $1 \leq i \leq k$,

c) if, again, $\widetilde{h}_{0}^{i} x \neq \widetilde{h}^{i} x$ for some $1 \leq i \leq k$, then $\sigma \cdot x \in W^{u}\left(x^{\prime}, H, U^{\prime}\right)$ for some $x^{\prime} \neq \sigma \cdot x, H x^{\prime}=x^{\prime}$ and 1-sided neighbourhood $U^{\prime}$ of $x^{\prime}$ in $I$, and $\sigma \in \pi_{1}\left(T_{1}\right)$.

To see a), note that if $V$ is any connected set in $\widetilde{T}, \widetilde{h}_{0} V \subset \widetilde{h} V$. We see b) as follows. If $x \in \widetilde{T}_{1}$ and $H^{\prime} x=x$ but $\widetilde{h}^{i} x \neq \widetilde{h}_{0}^{i} x$ for some $0<i \leq k$, then we can find inductively $x_{j} \in \widetilde{T}_{1}(0 \leq j<k)$ with $x_{0}=x, \widetilde{h} x_{j+1}=\widetilde{h}_{0} x_{j+1}=x_{j}$. 
Then for some $\zeta \in \pi_{1}\left(T_{1}\right), \zeta . x_{k} \neq x$ but $H\left(\zeta . x_{k}\right)=H^{\prime}\left(\zeta . x_{k}\right)=x$. Here, $\zeta$ is determined by $\tau$, and by the automorphism of $\pi_{1}\left(T_{1}\right)$ which is itself determined by the homotopy class of $\widetilde{h}$. Moreover, $W^{u}\left(x, H^{\prime}, U\right)$ is a point (for any 1 -sided neighbourhood $U$ of $x$ ), because, if $i$ is the least integer $>0$ with $\widetilde{h}^{i} x \neq \widetilde{h}_{0}^{i} x$, then $i$ is also the least integer $>0$ with $\widetilde{h}^{i} x \notin \widetilde{T}_{1}$, and there is a neighbourhood $V$ of $x$ with $\widetilde{h}_{0}^{i} V=\widetilde{h}_{0}^{i} x$, and then $H^{\prime} V=x$. Now for c): by (3.6) there is $x^{\prime} \in I$ and $\eta \in \pi_{1}\left(T_{1}\right)$ such that $\eta \cdot x_{k} \in W^{u}\left(x^{\prime}, H^{\prime}, U^{\prime}\right)$ for some 1 -sided neighbourhood of $x^{\prime}$ in $I$. Then $H\left(\eta \cdot x_{k}\right)=H^{\prime}\left(\eta \cdot x_{k}\right)=\sigma \cdot x \in W^{u}\left(x^{\prime}, H^{\prime}, U^{\prime}\right)$ for some $\sigma \in \pi_{1}\left(T_{1}\right)$. Now $\eta \cdot x_{k} \neq \sigma . x$ because $\widetilde{h}^{i}\left(\eta \cdot x_{k}\right) \in \widetilde{T}_{1}$ for $0 \leq i \leq k$, but this is not true for $\sigma \cdot x$, because it is not true for $x$. So $H^{\prime}\left(\eta \cdot x_{k}\right) \neq \eta \cdot x_{k}$ and $\eta \cdot x_{k} \neq x^{\prime}$. So $W^{u}\left(x^{\prime}, H^{\prime}, U^{\prime}\right)$ is not a point, so by b) $\widetilde{h}_{0}^{i} x^{\prime}=\widetilde{h}^{i} x^{\prime}$ for $0 \leq i \leq k$ and $W^{u}\left(x^{\prime}, H^{\prime}, U^{\prime}\right) \subset W^{u}\left(x^{\prime}, H, U^{\prime}\right)$ by a).

\section{7.}

There are two cases of the 1 -sided Neighbourhood Theorem to prove, corresponding to cases 1 and 2 of the Pseudo-Anosov Proposition. In case 2, recall that $\chi_{0}$ fixes a disc $D$ round $x_{0}$, and that $Y_{2}=Y_{1} \backslash D$. Then we choose a graph $T_{2} \subset Y_{2}$ to be of the form $\left(T_{1} \backslash D\right) \cup \partial D$, so that $T_{2} \hookrightarrow Y_{2}$ is a homotopy inclusion, and we choose maps $h_{2}: T_{2} \rightarrow T_{2}$ and $p_{2}: T_{2} \rightarrow T_{1}$ so that $p_{2} \circ h_{2}=h_{0} \circ p_{2}$, and $p_{2}$ is injective except for mapping $\partial D$ to $x_{0}$. Thus, $p_{2}$ extends to a map $Y_{1} \rightarrow Y_{1}$ homotopic to the identity, and $h_{2}$ is homotopic to $g \mid T_{2}$. Let $\widetilde{p}_{2}$ be any lift of $p_{2}$ to $\widetilde{Y}_{1}$. In case 2 , it suffices to prove

(3.7) for some closed arc $I$ in $\widetilde{T}_{2}$ projecting down to cover $T_{2}$, and some lift $H^{\prime}$ of $h_{2}^{k}, I \subset \bigcup\left\{W^{u}\left(x, H^{\prime}, U\right): x \in I, H^{\prime} x=x\right.$ and $U$ is a 1-sided neighbourhood of $x$ in $I\}$.

For suppose this is true. Then, by projecting by $\widetilde{p}_{2}$ we find that (3.6) is true also for a suitable $I$ and a suitable lift $H^{\prime}$ of $h_{0}^{k}$.

\subsection{A further reduction.}

We are now reduced to proving either (3.6) or (3.7), depending on whether we are in case 1 or 2 . We claim that it suffices to prove

(3.8) There is a closed arc $I$ in $\widetilde{T}_{1}$ (case 1) or $\widetilde{T}_{2}$ (case 2) which projects to cover all of $T_{1}$ (case 1) or $T_{2}$ (case 2) and such that $I$ has endpoints $a, b$, and there is a lift $H^{\prime}$ of $h_{0}^{k}$ (case 1) or $h_{2}^{k}$ (case 2) such that either $H^{\prime} a=a$ or $a$ separates $H^{\prime} a$ and $b$, and either $H^{\prime} b=b$ or $b$ separates $a$ and $H^{\prime} b$, so that, in particular, $I \subset H^{\prime} I$.

We shall complete the proof of the 1 -sided Neighbourhood Theorem by proving that (3.8) holds in both cases 1 and 2. For the justification of this claim, we use the following lemma. Part (i) shows that $I$ contains at least one fixed point. Then parts (ii) and (ii) are applied to subarcs of $I$ between fixed points. 
3.9. Lemma. Let $J$ be a closed arc in a tree with endpoints $a$, $b$, and let $H: T \rightarrow T$ be continuous. Then the following hold.

(i) If $H(a)$ is separated from $b$ by $a$, and $H(b)$ is separated from $a$ by $b$, then $J$ contains a fixed point of $H$.

(ii) If $H(a)=a$ and $H(b)$ is separated from $a$ by $b$, and $J$ has no fixed interior point, then $J \subset W^{u}(a, H, J)$.

(iii) If $H(a)=a, H(b)=b$ and $J$ has no fixed interior point, then $J \subset$ $W^{u}(a, H, J) \cup W^{u}(b, H, J)$.

Proof: (i) Inductively we can take $J=J_{0}$, and find intervals $J_{n+1} \subset J_{n}$ with $J_{n} \subset H\left(J_{n+1}\right)$, and such that either $J_{n}$ has a fixed endpoint for some $n$, or the conditions of (i) are satisfied by $J_{n}$ for all $n$. In this latter case, $\cap J_{n}$ is either an interval with fixed endpoints, or is a fixed point.

(ii) By (i), for $a^{\prime} \in \operatorname{Interior}(I)$ sufficiently near $a, a^{\prime}$ is always separated from $b$ by $H\left(a^{\prime}\right)$. Let $c$ be the endpoint of $W^{u}(a, H, J) \cap J$ in $J \backslash\{a\}$. If $c \neq b$, then $c$ separates $H(c)$ from $b$, since $H(c) \neq c$. By a), this cannot happen. So $c=b$. Then $b \in W^{u}(a, H, J)$, since $b \in H(\operatorname{Interior}(J)) \subset W^{u}(a, H, J)$ if $c=b$. So $J \subset W^{u}(a, H, J)$, as required.

(iii) By (i), we can assume without loss of generality that $a^{\prime}$ is separated from $b$ by $H\left(a^{\prime}\right)$ for $a^{\prime} \in \operatorname{Interior}(J)$ sufficiently near $a$. Let $c$ be the endpoint of $W^{u}(a, H, J) \cap J$ in $J \backslash\{a\}$. If $c=b$, we are done. If not, then as in (ii), $c$ separates $H(c)$ from $b$. Then we can complete the proof by applying (ii) with $J$ replaced by the interval between $c$ and $b$, and $a$ replaced by $b$ and $b$ replaced by $c$.

\subsection{Proof of (3.8) in Case 1.}

By choice of $\widetilde{h}$, we can ensure that $\widetilde{h}_{0}$ fixes a preimage of $x_{0}$ in $\widetilde{T}_{1}$. Take $H^{\prime}=\widetilde{h}_{0}^{k}$, and let the homotopy between $\chi_{0}^{k}$ and $h_{0}^{k}$ lift to one between a map $K$ and $H^{\prime}$. Then $K$ also fixes the same preimage of $x_{0}$, so $K=$ identity, since $\chi_{0}^{k} \mid Y_{1}=$ identity. So $H^{\prime}$ fixes all preimages of $x_{0}$. We can take $I$ to be any $\operatorname{arc}$ in $\widetilde{T}_{1}$ with endpoints at preimages of $x_{0}$ and projecting to cover $T_{1}$.

\subsection{Proof of (3.8) in Case 2.}

Let $\ell, x_{1}$ be as in case 2 of the Pseudo-Anosov Proposition. Let $M>0$, yet to be chosen. Let $\ell_{1}$ be a segment of $\ell$ with endpoint at $x_{1}$, such that

$$
\operatorname{length}\left(\chi_{0}^{k}\left(\ell_{1}\right) \backslash \ell_{1}\right)>M
$$

Let $\beta$ be the component of $\partial Y_{1}$ containing $x_{1}$. We can choose a lift $\widetilde{\beta}$ of $\beta$ in $\widetilde{Y}_{2}$ and a lift $K$ of $\chi_{0}^{k}$ so that all preimages of $x_{1}$ in $\widetilde{\beta}$ are fixed by $K$. Let $x_{1}^{\prime}, x_{1}^{\prime \prime}$ be two distinct preimages of $x_{1}$ in $\widetilde{\beta}$, let $\ell_{1}^{\prime}, \ell_{1}^{\prime \prime}$ be the lifts of $\ell_{1}$ with endpoints at $x_{1}^{\prime}, x_{1}^{\prime \prime}$, let $a^{\prime}, b^{\prime}$ be the second endpoints of $\ell_{1}^{\prime}, \ell_{1}^{\prime \prime}$, and let $J$ be 
the arc in $\ell_{1}^{\prime} \cup \ell_{1}^{\prime \prime} \cup \widetilde{\beta}$ between $a^{\prime}$ and $b^{\prime}$. Then $J \subset K(J), a^{\prime}$ separates $K\left(a^{\prime}\right)$ from $b^{\prime}, b^{\prime}$ separates $K\left(b^{\prime}\right)$ from $a^{\prime}$, and by choice of $M$ given $M_{1}>0$, we can ensure that

$$
d\left(K\left(a^{\prime}\right), a^{\prime}\right)>M_{1}, d\left(K\left(b^{\prime}\right), b^{\prime}\right)>M_{1} .
$$

Since $T_{2} \hookrightarrow Y_{2}$ is a homotopy equivalence, there is $q: Y_{2} \rightarrow T_{2}$ which is homotopic to the identity as a map from $Y_{2}$ to $Y_{2}$. Then the homotopy lifts to one between a map $\widetilde{q}$ and the identity. Now we choose $\ell_{1}$ so large that $q(J)=T_{2}$, which is possible by the Pseudo-Anosov Proposition. Then there is $M_{2}$ such that

$$
d\left(K x, H^{\prime} \widetilde{q} x\right) \leq M_{2} \text { for all } x \in \widetilde{Y}_{2},
$$

where the homotopy between $\chi_{0}^{k}$ and $h_{0}^{k}$ lifts to one between $K$ and $H^{\prime}$. We can choose $M_{1}>M_{2}$. Then the $\operatorname{arc} I$ between $a=\widetilde{q} a^{\prime}$ and $b=\widetilde{q} b^{\prime}$ satisfies (3.8), as required. 


\section{Chapter 4. The Nonrational Lamination Map Theorem.}

\section{1 .}

The aim of this chapter is to prove the following theorem. For the definitions of invariant lamination, lamination map and so on, see Chapter 6 of [R2]. The definition of primitive can be found in Chapter 7 of [R2], and is quite complicated, but can mostly be forgotten.

\section{The Nonrational Lamination Map Theorem.}

Let $\rho$ be a critically finite branched covering with a nondegenerate Levy cycle, which is also a lamination map, preserving a primitive invariant lamination $L$. Then there exist integers $m, k$, finite unions $A_{0} \subset A_{1} \subset \rho^{-1} A_{0}$ of leaf segments of $L$, and finite unions $B_{0}, B_{1} \subset \rho^{-1} B_{0}$ of $S^{1}$-segments, such that the following hold.

a) Either $m=1$, or $m k$ is less than the minimum period of a periodic critical point of $\rho$.

b) Both $A_{0} \cup B_{0}$ and $A_{1} \cup B_{1}$ have $m$ components, each homotopic in $\overline{\mathbf{C}} \backslash X(\rho)$ to a $k$-holed sphere with boundary components nontrivial in $\overline{\mathbf{C}} \backslash X(\rho)$.

c) Each component of $A_{0}$ contains a segment mapped into itself by $\rho^{m k}$, with orientation preserved.

d) For all $n \geq 0, \rho^{-n} B_{0} \subset S^{1}$ and diameters of components tend to 0 as $n \rightarrow \infty$.

e) The sets $A_{0} \cup B_{0}$ and $A_{1} \cup B_{1}$ are homotopic in $\overline{\mathbf{C}} \backslash X(\rho)$ via a homotopy which is constant on $A_{0}$, and homotopes each component of $\left(A_{1} \backslash A_{0}\right) \cup B_{1}$ into $B_{0}$.

4.2. Remark. If the theorem holds, we can inductively define $A_{n}$ with $A_{n} \subset$ $A_{n+1}$ and $A_{n+1}$ a union of components of $\rho^{-1} A_{n}$, and $A_{n}$ converges to a finite union $A$ of leaves, each of which is fixed by $\rho^{m k}$, and $\overline{\cup A}$ is noncontractible in $\overline{\mathbf{C}} \backslash X(\rho)$. It has $m$ components, unless $A$ contains compact geodesics.

\subsection{Outline of Proof.}

The theorem will be proved by choosing $Y$ for $\rho=g$ as in the conclusion of the Fixed Holed-Sphere Theorem with certain additional properties, and then applying the 1 -sided Neighbourhood Theorem to $Y$ and some embedded graph $T$, where the isotopy class of the homeomomorphism $\chi$ is given by a local inverse of $\rho^{m}$. Thus we have to choose $Y, T, h: T \rightarrow T$ with certain properties and deduce the theorem from this. 


\subsection{Recall of some basic definitions.}

We shall need to refer to [R2] to some extent, particularly Chapters 6 and 7 . Throughout the present chapter, $L$ is a $\beta$-invariant lamination on $\overline{\mathbf{C}} \backslash K$, with lamination map $\rho$, which therefore preserves $L$ and the Cantor set $K$, which is contained in $S^{1}$. Recall that a leaf segment is the closure of a component of $\ell \backslash S^{1}$, for a leaf $\ell$ of $L$. A polygon is the closure $P$ of a subset of $\overline{\mathbf{C}} \backslash S^{1}$ with $\partial P \subset(\cup L) \cup S^{1}$. A gap is a component of $\overline{\mathbf{C}} \backslash(K \cup(\cup L))$. A gap polygon is a polygon $P$ such that $\operatorname{Interior}(P)$ is a component of $G \backslash S^{1}$ for a gap $G$. A side of a gap $G$ is a leaf $\ell$ with $\ell \cap P \neq \phi$ for some gap polygon $P$ of $G$.

It was shown in [R2] - in particular, in 6.10 - that there are gap polygons $Q_{i}(1 \leq i \leq r)$ and points $a_{i}$ in $K(1 \leq i \leq q)$ having the following properties, and so that $\rho$ can be chosen to satisfy the following condition, given $\varepsilon^{\prime}>0$.

$$
\rho^{-n}\left(S ^ { 1 } \backslash \left(\left(\bigcup_{i=1}^{q} I_{i}\right) \cup\left(\bigcup_{i=1}^{r} \operatorname{Interior}\left(Q_{i}\right)\right) \subset S^{1} \text { for all } n \geq 0,\right.\right.
$$

where $a_{i}$ is strictly preperiodic under $\rho$, and $I_{i}$ is an interval in $S^{1} \backslash K$ of length $<\varepsilon^{\prime}$ and with endpoint at $a_{i}$, and $\rho\left(\cup Q_{i}\right) \subset \cup Q_{i}$. We shall need this because we shall need to consider families of leaf segments and $S^{1}$-segments, which we shall want mapped to unions of leaf segments and to $S^{1}$-segments by inverse images of $\rho$.

\subsection{Essential Leaf Segments.}

For suitable $\zeta>0$, we say that a leaf segment $\ell$ is essential if some components of $\rho^{-n} \ell$ have diameter $>\zeta$ for infinitely many $n$. By the Length Lemma ([R2], 6.15), for suitable $\zeta$, this is only possible if either

diameter $(\ell)>\zeta$, or

diameter $(\ell) \leq \zeta$ and $\ell$ has endpoints on opposite sides of, but close to, some $a_{i}$ $(1 \leq i \leq q)$.

From the definition (and for suitable $\zeta$ ), if $\ell$ is not essential, then all components of $\rho^{-n} \ell$ are nonessential leaf segments, for all $n \geq 0$.

\subsection{Properties of $Y$.}

As we have said, we need to construct $Y, T, h$. We summarise the properties of $Y$ we shall need, and which are proved in 4.7-4.10. That the first property is possible is simply a restatement of part of the Fixed Holed-Sphere Theorem.

1. $Y$ is an open $\ell$-holed sphere in $\overline{\mathbf{C}} \backslash X(\rho)$ for some $\ell \geq 2$, with each boundary component nontrivial, and for some local inverse $S$ of $\rho^{m}, Y$ and $S Y$ are isotopic in $\overline{\mathbf{C}} \backslash X(\rho)$, and $S$ cyclically permutes boundary components up to isotopy in $\overline{\mathbf{C}} \backslash X(\rho)$. 
2. Either (i) or (ii) holds, depending on whether the Expanding Fixed Set or Nonrational Adjacent case of the Fixed Holed-Sphere Theorem holds.

(i) $m=1$ and $S Y \subset Y$.

(ii) Here, $m$ is such that $m \ell$ less than the minimum period of a periodic critical point of $\rho$, and $\partial Y=\partial_{1} Y \cup \partial_{2} Y$, where $S \bar{Y} \cap \partial_{1} Y=\phi$, and $S \partial_{2} Y \cap \bar{Y}=\phi$. Each component $C$ of $S Y \backslash Y$ intersects only one component of each of $\partial_{2} Y$, $S \partial_{2} Y$.

For (i) see 4.7, and (ii) is a restatement of the Fixed Holed-Sphere Theorem.

3. Either (i) or (ii) holds, depending, again, on the case of the Fixed Holed-Sphere Theorem.

(i) $\partial Y$ is a finite union of leaf segments and $S^{1}$-segments.

(ii) $\partial_{1} Y$ is a finite union of leaf segments and $S^{1}$-segments, $\partial_{2} Y$ is a finite union of $S^{1}$-segments.

For (i), see 4.7. For (ii), see 4.9.

Note. We shall write $S^{n} Y$ even when $S Y \backslash Y \neq \phi$, meaning the local inverse defined on $S^{n-1} Y$ by lifting the inductively defined homotopy between $S^{n-2} Y$ and $S^{n-1} Y$.

4. $S^{i} Y \cap\left\{a_{1} \ldots a_{q}\right\}=\phi$ for all $i \geq 0$, and if $b$ is a component of $\partial Y \cap S^{1}$, then $b \cap \bigcup_{i=1}^{r} Q_{i}=\phi$ so that $S^{n} b \subset S^{1}$ for all $n$.

See 4.7 .

5 .

$$
\bar{Y}=\bigcup_{i=1}^{v} Y_{i}
$$

where the sets Interior $\left(Y_{i}\right)$ are disjoint, and, for some $0 \leq t \leq u \leq v$, the following hold.

a) $\partial Y_{i} \backslash \partial Y \subset S^{1}$, and if $b$ is a component of $\partial Y_{i} \cap S^{1}, b \cap \bigcup_{i=1}^{r} Q_{i}=\phi$, so that $S^{n} b \subset S^{1}$ for all $n$.

b) For $i>t, Y_{i}$ is a single polygon not containing any periodic gap polygon such that $\partial Y_{i}$ contains exactly two essential leaf segments for $t<i \leq u$, and none for $u<i \leq v$.

c) For $i \leq t, Y_{i}$ is a finite connected union of polygons, and there is a finite connected union $Y_{i}^{\prime} \subset Y_{i}$ of gap polygons such that each maximal polygon of $Y_{i}$ contains exactly one polygon of $\left.Y_{i}^{\prime},\left(Y_{i} \backslash Y_{i}^{\prime}\right) \cap S^{1}\right) \cap \bigcup_{i=1}^{r} Q_{i}=\phi$, and there is a bijection $\tau:\{1 \ldots t\} \rightarrow\{1 \ldots t\}$ with

$$
Y_{\tau(i)}^{\prime} \subset S Y_{i}^{\prime} .
$$

d) $S Y_{j} \cap Y_{i}=\phi$ for $i, j \leq t$ and $i \neq \tau(j)$, and $S Y_{j} \cap Y_{\tau(j)}$ is connected.

See 4.10 (and 4.7-4.9). 


\subsection{Lemma.}

$Y$ can be chosen so that either 1 or 2 holds, where $S$ is a local inverse of $\rho^{m}$.

1. $m=1, \partial Y$ is a finite union of leaf segments and $S^{1}$-segments, and $S Y \subset Y$.

2. $\partial Y=\partial_{1} Y \cup \partial_{2} Y$, where $\partial_{i} Y$ is a finite union of leaf segments and $S^{1}$

-segments,

$$
S \bar{Y} \cap \partial_{1} Y=\phi, S \partial_{2} Y \cap \bar{Y}=\phi,
$$

and each component of $S Y \backslash Y$ has one component of intersection with each of $\partial_{2} Y, S \partial_{2} Y$.

Furthermore, we can ensure that $\partial Y \cap S^{1} \cap Q_{i}=\phi(1 \leq i \leq r)$ and $S^{n} Y \cap\left\{a_{1} \ldots a_{q}\right\}=\phi$ for all $n \geq 0$.

Proof:

Suppose the Expanding Fixed Set case of the Fixed Holed-Sphere Theorem holds. Then the variation of 2.3 implies $Y$ can be chosen to contain all leaves and finite-sided gaps of $L$ it intersects, and so that $S Y \subset Y$ and $S^{p} \bar{Y} \subset Y$ for some $p$. For any finite connected union of leaf segments, or arc in a finitesided gap has the property of $\gamma$ in the statement of the variation. Then we can approximate $S^{i} Y(0 \leq i<p)$ by a set $Y_{i}$ whose boundary is a finite union of leaf segments, components of intersection with gap polygons in infinite-sided gaps and their sides, and $S^{1}$-segments, such that $\bar{Y}_{i+1} \subset Y_{i}$ for $i \leq p-2, S \bar{Y}_{p-1} \subset Y_{0}$ and $\partial Y_{i} \cap \partial Q_{j} \cap S^{1}=\phi$. Then $Y^{\prime}=\cap_{i=0}^{p-1} Y_{i}$ satisfies $S \bar{Y}^{\prime} \subset Y^{\prime}$. Let $Y$ be obtained from $Y^{\prime}$ by removing all components of intersection with gap polygons in infinite-sided gaps and their sides which are not complete gap polygons. Then $S Y \subset Y$. We can ensure $S^{n} Y \cap\left\{a_{1} \ldots a_{q}\right\}=\phi$ for all $n \geq 0$ by replacing $Y$ by $S^{n} Y$ for a suitable $n \geq 0$, because the $a_{i}$ are strictly preperiodic.

Suppose the Nonrational Adjacent case of the Fixed Holed-Sphere Theorem holds. Then the variations of 2.3 ensure that we can choose $Y$ so that $\partial_{1} Y, \partial_{2} Y$ consist of finitely many leaf and $S^{1}$-segments, and do not intersect $S^{1} \cap Q_{i}$ for any $i$. Then $\partial_{1} Y, \partial_{2} Y$ have all the required properties, provided, as in case 1, we replace $Y$ by $S^{n} Y$ if necessary.

\subsection{Lemma.}

$Y$ can be chosen so that the periodic gap polygons contained in $S^{n} Y$ are the same for all $n \geq 0$, and such that no two periodic gap polygons $P, Q$ in $Y$ lie in the same component of $Y \backslash S^{1}$.

Proof: Since the lamination $L$ is primitive, the total number of periodic gap polygons is finite. (See 6.15, 7.2 and 7.3 of [R2]. A periodic thin gap polygon lies in a periodic gap bounded by periodic segments on some of the finitely many periodic sides. But in a primitive lamination, there is a bound on the number of periodic segments on any leaf.) If $S Y \subset Y$, the number of periodic gap polygons 
in $S^{n} Y$ obviously decreases with $n$, so replacing $Y$ by a suitable $S^{n} Y$, we can assume the number is always constant. If $Y$ satisfies the Nonrational Adjacent case of the Fixed Holed-Sphere Theorem, so that $S Y \backslash Y \neq \phi$, let $p$ be the maximal period of a periodic gap polygon. If some periodic gap polygon is in $S Y \backslash Y$, then for some $1 \leq n \leq p, S^{n}(S Y \backslash Y) \cap Y \neq \phi$ - but, by the variations of 2.3 of the Fixed Holed-Sphere Theorem, $Y$ can be chosen so that this does not happen.

Finally, suppose for contradiction that $P, Q$ are in the same component of $S^{n} Y \backslash S^{1}$ for all $n$. Then $P, Q$ are finite-sided, since $S^{n} \mid Y$ is a homeomorphism for all $n$. We can assume there are no periodic gap polygons between $P$ and $Q$ in the same component of $Y \backslash S^{1}$. But the region between $P$ and $Q$ must be mapped back into itself by some $S^{n}$, so it must be a single periodic leaf segment, which is impossible, because gaps are never immediately adjacent in a primitive lamination. So, again, replacing $Y$ by $S^{n} Y$ if necessary, $Y$ has the required properties.

\subsection{Lemma.}

In the case when $\partial Y=\partial_{1} Y \cup \partial_{2} Y, Y$ can be chosen so that $\partial_{2} Y \subset S^{1}$.

Proof: Let $C$ be a component of $S Y \backslash \bar{Y}$. Then by the variations of 2.3, the distance between $\bar{C} \cap \partial_{2} Y$ and $\bar{C} \cap S \partial_{2} Y$ can be assumed so large that no maximal polygon in $C$, or union of polygons intersecting some $Q_{i}$, has sides in both $\partial_{2} Y$ and $S \partial_{2} Y$. Then we can find an $S^{1}$-arc in $C$, which intersects no $Q_{i}$, separating $\bar{C} \cap \partial_{2} Y$ and $\bar{C} \cap S \partial_{2} Y$. Then we replace $Y$ by $S Y$ cut off by $S^{1}$-arcs in all such components $C$.

\subsection{0.}

4.7 to 4.9 show that $Y$ can be chosen satisfying 1 to 4 of 4.6 , and part of 5 of 4.6: we can write $\bar{Y}=\cup_{i=1}^{r} Y_{i}$ so that $Y_{i}$ is a single polygon not containing a periodic gap polygon for $i>t$, and $Y_{i}$ has the properties listed in 5 of 4.6 for $i \leq t$, except for 5b) and 5d). Now we claim that, if we replace $Y$ by $S^{n} Y$ for a suitable $n \geq 0$, and rename and renumber the $Y_{i}$, but keeping the name $Y_{i}$ $(i \leq t)$ for the union of polygons of $\bar{Y}$ intersecting $Y_{i}^{\prime}$, then 5 of 4.6 is satisfied in its entirety. For gap polygons are dense and all eventually periodic, so we can ensure that, for $i>t, Y_{i}$ does not contain any specified gap polygon, by choosing $n$ large enough. So then, given $\zeta>0$, we can ensure that $\partial Y_{i}$ has at most two leaf segments of length $>\zeta / 2$, and if at most one has length $>\zeta / 2$, they all have length $<\zeta$. Thus we can ensure that $\partial Y_{i}$ contains either exactly two essential leaf segments, or none, for $i>t$, and, renumbering, 5a)-c) of 4.6 are satisfied. we can also assume that $\cup_{i=1}^{t}\left(Y_{i} \backslash Y_{i}^{\prime}\right) \cap \cup_{i=1}^{t} S Y_{i}^{\prime}=\phi$, so that $5 \mathrm{~d}$ ) is also satisfied, as required. 


\subsection{Properties of $\mathrm{U}, \mathrm{U}^{\prime}$.}

Let $U \subset \bar{U} \subset U^{\prime}$ be open sets in $\bar{Y}$ satisfying the following - which are possible.

a)

$$
\bigcup_{i>u} Y_{i} \subset U
$$

and, in the Nonrational Adjacent case,

$$
\partial_{2} Y \subset U
$$

b) For $t<i<u, Y_{i} \cap U$ has two components, and $\partial\left(Y_{i} \backslash U\right) \backslash \partial U$ has two components, which contain $\bar{Y}_{i} \cap S^{1}$, and the essential leaf segments in $\partial Y_{i}$ run between these two components.

c) For $i \leq t$, each component of $Y_{i} \cap U$ contains exactly one component of $\partial Y_{i} \cap S^{1}$.

d) Similar properties to a), b) and c) hold for $U^{\prime}$.

e)

$$
S \bar{U} \cap Y \subset U^{\prime} .
$$

f) Each component of $S Y \backslash Y$ intersects a unique component of $U^{\prime}$.

\subsection{Properties of $T$.}

We can define a graph $T \subset Y$ satisfying the following.

a) $T \hookrightarrow Y$ is a homotopy equivalence.

b) $T$ has no vertex in $Y_{i} \backslash U$ for $t<i \leq u$, but has one edge crossing between the components of $U$ bounding $Y_{i}$.

c) For $i \leq t, T \cap Y_{i} \subset Y_{i}^{\prime}, T$ has exactly one vertex $v_{i}$ in $Y_{i}^{\prime} \backslash U$ with $S v_{i} \in$ $Y_{\tau(i)}^{\prime} \backslash U^{\prime}$, and exactly one edge of $T$ runs from $v_{i}$ into each bounding component of $U$.

d) No arc of $S T \backslash U^{\prime}$ has both endpoints in the same component of $U$.

\subsection{Definition of $\pi, \mathrm{h}$.}

We can define $\pi: S T \rightarrow T$ satisfying the following.

a) $\pi$ maps each component of $S T \cap U \backslash \cup_{i=1}^{t} Y_{i}$ into the component of $U^{\prime}$ it is contained in, and each component of $S T \backslash Y$ into the component of $U^{\prime}$ it intersects.

b) $\pi$ maps $S T \backslash\left(U^{\prime} \cup \bigcup_{i=1}^{t} Y_{i}\right)$ to $T \backslash U$, and is monotone restricted to each arc in $S T \backslash\left(U \cap \bigcup_{i=1}^{t} Y_{i}\right)$.

c)

$$
\pi S v_{i}=v_{\tau(i)} .
$$


d) Any component of $S T \cap\left(\bigcup_{i=1}^{t} Y_{i} \backslash U\right)$ whose closure intersects only one component of $U^{\prime}$ is mapped by $\pi$ into that component of $U^{\prime}$. For $i \leq t, \pi$ is monotone restricted to each arc in $S T \cap Y_{i} \backslash U^{\prime}$ which has endpoints in different components of $U^{\prime}$, and maps it to an arc in $Y_{i} \cap T$ with endpoints in the same components of $U$.

Then we define

$$
h=\pi \circ S
$$

\subsection{Properties of $h$.}

From the definition of $h$, we obtain the following.

$$
h(U) \subset U \text {. }
$$

b) If $t<j \leq u$ and $i \leq u$, then $h\left(T \cap Y_{i} \backslash U\right)$ contains $T \cap Y_{j} \backslash U$ if it intersects it, This happens if and only if $S Y_{i} \cap Y_{j}$ separates the essential leaf segments in $\partial Y_{j}$.

c) For $i \leq t, h\left(v_{i}\right)=v_{\tau(i)}$, and if $j \leq t$ also,

$$
h\left(T \cap Y_{i} \backslash U\right) \cap Y_{j}=\phi,
$$

unless $j=\tau(i)$, when $h^{-1}\left(Y_{j} \cap T \backslash U\right) \cap T \cap Y_{i} \backslash U$ is connected, and then, if $b$ is a component of $Y_{i} \backslash U \backslash\left\{v_{i}\right\}, h(b)$ contains exactly one component of $Y_{j} \backslash U \backslash\left\{v_{j}\right\}$ and intersects no other.

Now the 1-sided Neighbourhood Theorem will imply the existence of $k$ with $2 \leq k \leq \ell$ and a subgraph $T_{1}$ of $T$ with

d) $\quad T_{1} \backslash U \subset \bigcup\left\{W^{u}\left(x, h^{k}, V\right): x \in T_{1} \backslash U, h^{i} x \in T_{1}\right.$ for all $i, h^{k} x=x$,

$V$ is a 1 -sided neighbourhood of $x$ in $\left.T_{1}\right\}$.

Then the properties of $\pi$ imply that

e) if $h^{k} x=x$ and $W^{u}\left(x, h^{k}, V\right) \neq \phi$ for some $x \in T_{1} \cap Y_{i} \backslash U$, some $i \leq t$ and for some $V$, then $b \subset S^{k} b$ for each component $b$ of $\partial Y_{i}^{\prime} \cap(\cup L)$. Moreover, given $i \leq t$, there must be such an $x, V$, unless every $\operatorname{arc}$ of $T_{1} \cap Y_{i} \backslash U^{\prime}$ is in $h\left(T_{1} \backslash Y_{i}\right)$, that is, unless, for any maximal arc $b$ of $T_{1} \cap Y_{i}$, there is an arc of $S\left(T_{1} \cap Y \backslash Y_{i}\right) \cap Y_{i}$ running parallel to it throughout its length.

\subsection{Proof of the Nonrational Lamination Map Theorem.}

We shall find $A_{0}$ such that exactly one connected leaf piece from $A_{0}$ runs parallel to each arc in $T_{1} \cap\left(\bigcup_{i=1}^{u} Y_{i} \backslash U\right)$. Then we shall join the endpoints of the leaf pieces of $A_{0}$ by $S^{1}$-arcs whose union we denote by $B_{0}$, so that $A_{0} \cup B_{0}$ is homotopic to $T_{1}$ in $\overline{\mathbf{C}} \backslash X(\rho)$. We shall have $\rho^{-n} B_{0} \subset S^{1}$ for all $n$, since each component of $B_{0}$ is a union of a subset of $\cup Y_{i} \cap S^{1}$ and of intervals in $S^{1}$ between points of $Y_{j} \cap S^{1}(j>u)$. Thus, $\rho^{-n} B_{0} \cap\left(\cup Q_{i} \cup\left\{a_{1} . . a_{q}\right\}\right)=\phi$ for all 
$n \geq 0$. The homotopy between $A_{0} \cup B_{0}$ and $A_{1} \cup B_{1}$ is obtained by constructing a homotopy between $A_{0} \cup B_{0}$ and $T_{1}$ which preserves $U, Y \backslash U$, constructing a similar homotopy between $S T_{1}$ and $A_{1} \cup B_{1}$, and constructing yet another similar homotopy between $\pi$ and the identity. So it remains to construct $A_{0}$. From properties b), d), e) of $h$ in 4.14, the proof of the theorem will be completed after the following lemma.

\subsection{Lemma.}

If $t<i \leq u$ and $x \in Y_{i} \cap T_{1} \backslash U, h^{k} x=x, h^{j} x \in T_{1}$ for $0 \leq j \leq k$, and $W^{u}\left(x, h^{k}, V\right) \neq \phi$, then there is a leaf segment $\ell$ in $Y_{i}$ separating the essential leaf segments in $\partial Y_{i}$ such that $\ell \subset S^{k} \ell$, and $S^{k} \mid \ell$ preserves orientation.

Proof:

Let $x \in Y_{i} \cap T_{1} \backslash U, t<i \leq u, h^{k} x=x, h^{j} x \in Y_{i_{j}} \cap T_{1} \backslash U$ with $i_{0}=i_{k}=i$. Then $t<i_{j} \leq u$ for all $j$. Inductively, let $Q_{0}=Y_{i}$, and let $Q_{j+1}$ be the component of $S Q_{j} \cap Y_{i_{j+1}}$ in the component of $S Y_{i_{j}} \cap Y_{i_{j+1}}$ which contains $S h^{j} x$ for $0 \leq j<k$. Then, for $n \geq k$, let $Q_{n+1}$ be the unique component of $S Q_{n} \cap Q_{n+1-k}$. Then inductively, using property b) of $h$ in 4.13 , we see that $Q_{n k+j}$ separates the essential leaf segments in $\partial Y_{i_{j}}$. Then $\cap Q_{n k}$ must be a leaf segment $\ell$ with $\ell \subset S^{k} \ell$, because otherwise it is a periodic gap polygon in $Y_{i}$, and there are none. If $W^{u}\left(x, h^{k}, V\right) \neq \phi$ for some $V$, then $S^{k} \mid \ell$ preserves orientation. This is because, for all $j \leq k$, the images under $S$ and $h$ of the endpoints of the arc of $T \cap Y_{i_{j}} \backslash U$ containing $h^{j} x$ lie in the same components of $U$, and the endpoints of the arc of $S^{j+1} \ell$ in $Q_{j+1}$ also lie in these same components of $U$.

\subsection{End of proof of the Theorem.}

It suffices to choose $A_{0} \subset Y$ so that a leaf segment from $A_{0}$ intersects $Y_{i} \backslash U$ for all $t<i \leq u$, and some connected union of leaf segments in $A_{0}$ runs parallel to each component of $\partial Y_{i}^{\prime}$, for all $i \leq t$. Now fix $i \leq u$, and suppose $W^{u}\left(x, h^{k}, V\right) \neq \phi$ for some $x \in T_{1} \cap Y_{i} \backslash U$, and $V$. If $i \leq t$ let $\partial Y_{i}^{\prime} \cap(\cup L) \subset A_{0}$, and if $t<i \leq u$, let $A_{0}$ include a leaf segment $\ell$ as in the lemma, and let $A_{0}$ also contain a finite connected union of segments containing $\ell$ which is contained in $Y$, has endpoints in $U$, and runs parallel to as many arcs of $T_{1} \backslash U$ as possible. Then $A_{0}$ has the required properties. 
Chapter 5. Proofs of the Admissible Boundary Theorem and the Admissibility Proposition.

5.1 .

The theorems to be proved in this chapter are stated in Chapter 1 of [R2], and will be restated here, but it will not be possible to explain all the terms involved, for which the reader should refer to Chapters 6 and 7 of [R2], in particular, for a full account of invariant and parameter laminations. The main result of the last chapter, the Nonrational Lamination Map Theorem, was essentially a result about invariant laminations (or their lamination maps). The task, now, is to transfer this to a result about parameter laminations.

\subsection{Invariant sets of complete geodesics.}

We recall, at least, that an invariant lamination $L$ is a geodesic lamination on $\overline{\mathbf{C}} \backslash K$, where $K$ is a Cantor subset of $S^{1}$, where $K=K_{r}$ depends on a parameter $r$ (which is an odd denominator rational). Also, there is a fixed lamination called (somewhat incongruously at this stage) $\bar{L}_{r}$ such that $L \cup \bar{L}_{r}$ is always a lamination, and $L$ contains all but countably many leaves of $\bar{L}_{r}$. The sets $S^{1}, K$ and $\bar{L}_{r}$ are invariant under a critically finite degree two branched covering $\bar{s}=\bar{s}_{r}$ which fixes $\infty$. For precise definitions of $K, \bar{L}_{r}, \bar{s}$, see Chapter 1 of $[\mathbf{R 2}]$.

The invariance is actually $\beta$ - invariance for some path $\beta:[0,1] \rightarrow \overline{\mathbf{C}} \backslash(K \cup$ $\left(\cup \bar{L}_{r}\right)$ ), and uses a homeomorphism $\sigma_{\beta}$ defined in terms of $\beta$. (See [R2] 1.7.) We recall that, in [R2] 6.5 we put extra conditions on $\beta$ in order to ensure that, for a complete geodesic $\ell$ in a $\beta$-invariant lamination, $\left(\sigma_{\beta} \circ \bar{s}\right)^{-1} \ell$ could be easily modified to a set of geodesics. We also need to do something similar here for finite geodesics, that is, geodesics with finitely many $S^{1}$-crossings, which may be incomplete. This means making modifications to $\beta$ which are similar to those of 6.5 of [R2], but slightly less technical. We describe these now.

\subsection{The Minimal Intersection Property.}

Let $\beta:[0,1] \rightarrow \overline{\mathbf{C}} \backslash\left(K \cup\left(\cup \bar{L}_{r}\right)\right)$ with $\beta(0)=\infty$ and let $A$ be a union of finite disjoint geodesic arcs in $\overline{\mathbf{C}} \backslash\left(K \cup\left(\cup \bar{L}_{r}\right)\right)$ with any endpoints in $S^{1}$. Then after isotopy in $\overline{\mathbf{C}} \backslash\left(K \cup\left(\cup \bar{L}_{r}\right)\right)$ keeping $\beta(0)=\infty$ and fixing $\beta(1), \beta$ can be assumed to have the following properties.

a) If $c, d$ are adjacent points of $\beta^{-1}\left(S^{1}\right)$, then $\beta(c), \beta(d)$ lie in different components of $S^{1} \backslash K$, and $\beta$ is transverse to $S^{1}$.

b) No sub-path of $\beta$ can be homotoped into $A$ by a homotopy in $\overline{\mathbf{C}} \backslash K$ fixing the sub-path endpoints, $\beta$ is transverse to $A$, and the number of points of 
$(0,1) \cap \beta^{-1}(A)$ cannot reduced by homotopies fixing $\beta(1)$ and $\beta(0)=\infty$, while keeping property a).

Let $\beta_{u}$ be any homotopy through paths in $\overline{\mathbf{C}} \backslash\left(K \cup\left(\cup \bar{L}_{r}\right)\right)$ satisfying a) and b) such that $\beta_{0}=\beta, \beta_{u}(0)=\beta(0)=\infty$ and $\beta_{u}(1)=\beta(1)$ for all $u$, and such that $\beta_{u}(t) \in A$ only if $\beta(t) \in A$. Now we can state condition c).

c) If $\beta(t) \in A$, then $\beta(t) \notin S^{1}, \beta_{u}(t) \in A$ and

$$
\#\left\{v<t: \beta(v) \in S^{1}\right\} \leq \#\left\{v<t: \beta_{u}(v) \in S^{1}\right\}
$$

for all $u$.

The conditions b), c) subject to a) ensure that $\left(\sigma_{\beta} \circ \bar{s}\right)^{-1} A$ has the least possible number of intersections with $S^{1}$, up to homotopy fixing the endpoints of $\left(\sigma_{\beta} \circ \bar{s}\right)^{-1} A$ and preserving $K$ and $\bar{L}_{r}$.

Then $(\beta, A)$ has the minimal intersection property if conditions a) to c) hold. A good isotopy will be one which preserves $S^{1}, K$ and $\bar{L}_{r}$.

Note the following.

1. The image of $(A, \beta)$ under any good isotopy also has the minimal intersection property.

2. If $A$ can be isotoped into $A^{\prime}$ by a good isotopy, then there is $\beta^{\prime}$ such that $\left(A^{\prime}, \beta^{\prime}\right)$ has the minimal intersection property, and such that $(A, \beta)$ can be isotoped into $\left(A^{\prime}, \beta^{\prime}\right)$ by a good isotopy.

3. For any finite set $A$ of finite geodesics in a $\beta$-invariant lamination with $\beta$ satisfying the conditions of 6.5 of $[\mathbf{R 2}],(A, \beta)$ has the minimal intersection property.

5.4. First Invariant-implies-Parameter Theorem. In what follows, all inclusions and equalities are up to good isotopy. Let $\left(A_{0}, \beta\right)$ have the minimal intersection property, with $\beta(1)$ in a component $\mu_{0}$ of $A_{0}$, and suppose that

$$
A_{0} \subset\left(\sigma_{\beta^{\prime}} \circ \bar{s}\right)^{-1} A_{0},
$$

where $\beta^{\prime}$ is an arbitrarily small extension (or restriction) of $\beta$. If $x \notin K$ is an endpoint of $A_{0}$, let $\tau(x)$ be the endpoint (if it exists) of $A_{0}$ such that $\left(\sigma_{\beta^{\prime}} \circ \bar{s}\right)^{-1}(\tau(x))$ is isotoped to $x$ by the good isotopy. Suppose also that, for any endpoint $x \notin K, \tau^{n}(x)$ is not defined for all $n$.

Then there are $\gamma$, and a $\gamma$-invariant lamination $L$, such that $A_{0} \subset L, \mu_{0}$ is contained in the minor leaf or a side of the minor gap of $L$, and $\left(A_{0}, \beta^{\prime}\right)$ or $\left(A_{0}, \beta\right)=\left(A_{0}, \gamma\right)$ up to good isotopy, depending on whether or not $L$ has a minor gap.

The minor leaf of $L$ is eventually periodic, and strictly preperiodic if $L$ does not have a minor gap. Moreover, no half leaf of $L$ has infinitely many successive intersections with periodic components of $S^{1} \backslash K$. 
Proof: Again, all inclusions and equalities are up to good isotopy. Write $\beta_{0}=\beta^{\prime}$. Inductively, define $\beta_{n}, A_{n}$ for $n \geq 1$ with the following properties.

1. $A_{n+1}=\left(\sigma_{\beta_{n}} \circ \bar{s}\right)^{-1} A_{n}$

2. $\left(A_{n}, \beta_{n}\right) \subset\left(A_{n+1}, \beta_{n+1}\right)$

3. $\left(A_{n}, \beta_{n}\right)$ has the minimal intersection property.

We can clearly define $A_{1}, \beta_{1}$. Suppose $A_{k}, \beta_{k}$ have been defined with the correct properties for $k \leq n$ and $n \geq 1$, and define $A_{n+1}$ by 1 . Then since $\left(A_{n-1}, \beta_{n-1}\right) \subset\left(A_{n}, \beta_{n}\right)$, we have $\left(\sigma_{\beta_{n-1}} \circ \bar{s}\right)^{-1} A_{n-1} \subset\left(\sigma_{\beta_{n}} \circ \bar{s}\right)^{-1} A_{n}$, and hence $A_{n} \subset\left(\sigma_{\beta_{n}} \circ \bar{s}\right)^{-1} A_{n}$. Then by 5.3.1 and 2 we can find a $\beta_{n+1}$ so that $\left(A_{n}, \beta_{n}\right) \subset\left(A_{n+1}, \beta_{n+1}\right)$.

Then the condition on $\left\{\tau^{n}(x)\right\}$ implies that, if $x$ is an endpoint of some $A_{n}$, then either $x \in K$ or $x$ is interior to $A_{m}$ for some $m>n$. It follows that $A_{n}$ converges to a lamination $L$, containing $A_{0}$. We can also assume that $\beta_{n}$ converges to $\gamma^{\prime}$. Here, $\gamma^{\prime}$ might contain points of $K$ which are endpoints of intervals of $S^{1} \backslash K$, but can be perturbed to $\gamma$ in $\overline{\mathbf{C}} \backslash(K \cup(\cup \bar{L}))$ such that $L$ is $\gamma$-invariant. Note that $A_{0}$ contains all the periodic segments in the full orbit of $A_{0}$, and $L$ is the closure of this full orbit. Hence no half leaf in $L$ has an infinite succession of periodic segments, as required.

Let $\ell_{0}$ be the component of $A_{0}$ containing $\beta(1)$ and let $\ell_{i+1}$ be defined inductively as a component of $A_{i+1}$ which is a component of $\left(\sigma_{\beta_{i}} \circ \bar{s}\right)^{-1} \ell_{i}$. Obviously, $\ell_{0}$ in $L$ is always in an eventually periodic leaf, and in a periodic leaf if and only if $\ell_{0} \subset \ell_{n}$ with orientation preserved for some $n$, for some choice of $\left\{\ell_{i}\right\}$. If this happens, no other component $\ell_{n}^{\prime}$ of $A_{n}$ can run parallel to $\ell_{n}$ for the whole length of $\ell_{n}$ on the same side as $\beta_{n}(1)$. For if so, let $\ell_{i}^{\prime} \quad(0 \leq i \leq n)$ be defined similarly to $\ell_{i}$, so that $\ell_{i+1}^{\prime}$ is a component of $\left(\sigma_{\beta_{i}} \circ \bar{s}\right)^{-1} \ell_{i}^{\prime}$. Suppose $\ell_{i+1}$ and $\ell_{i+1}^{\prime}$ run parallel along their entire lengths. Then $\sigma_{\beta_{i}}^{-1} \ell_{i}$ and $\sigma_{\beta_{i}}^{-1} \ell_{i}^{\prime}$ run parallel, and so do $\ell_{i}$ and $\ell_{i}^{\prime}$. Then, by induction, $\sigma_{\beta^{\prime}}^{-1} \ell_{0}$ and $\sigma_{\beta^{\prime}}^{-1} \ell_{0}^{\prime}$ run parallel along their entire lengths. This is impossible, because $\beta^{\prime}(1)$ separates $\ell_{0}$ and $\ell_{0}^{\prime}$. It follows that $\beta_{k}$ can be chosen so that $\left(A_{k}, \beta_{k}\right)$ converges to $\left(L, \gamma^{\prime}\right)$, modifying to $(L, \gamma)$, with $\gamma(1)$ in a minor gap of $L$. Thus, $L$ has all the required properties.

\section{5 .}

We recall that, in [R2], $\widetilde{U}$ denoted the universal cover of the union of component of $\overline{\mathbf{C}} \backslash\left(K \cup\left(\cup \bar{L}_{r}\right)\right)$ containing $\infty$ and some boundary leaves, with $0 \in \widetilde{U}$ projecting to $\infty$. If $\beta:[0,1] \rightarrow \overline{\mathbf{C}} \backslash\left(K \cup\left(\cup \bar{L}_{r}\right)\right)$ is a path with $\beta(0)=\infty$, then $\widetilde{\beta}$ denotes the lift to $\widetilde{U}$ of $\beta$ with $\widetilde{\beta}(0)=0$. Given a $\beta$-invariant lamination $L$ with minor gap, the lift to $\widetilde{U}$ containing $\widetilde{\beta}(1)$ is also called the minor gap, and similarly for minor leaves: the minor leaf in $\widetilde{U}$ either contains $\widetilde{\beta}(1)$, or is intersected by $\widetilde{\beta}$ and in the boundary of the minor gap, if this exists. 
We recall the Parameter Laminations Theorem (1.16 of [R2]) which says that minor leaves and boundaries of minor gaps of primitive invariant laminations never intersect transversally. Primitivity is no real restriction. As in the statement and proof of the First Invariant-implies-Parameter Theorem, inclusions and equalities for sets of geodesic pieces are up to good isotopy, in the following theorem.

\section{Second Invariant-implies-Parameter Theorem.}

Let $\left(A_{0}, \beta\right)$ and $\beta^{\prime}$ be as in the First Invariant-implies-Parameter Theorem. Let $L^{\prime}$ be a primitive $\gamma^{\prime}$-invariant lamination and let $\widetilde{\gamma}^{\prime}$ be the lift of $\gamma^{\prime}$ with $\widetilde{\gamma}^{\prime}(0)=0$. Suppose that there is an isotopy preserving $K, \bar{L}_{r}$ and $A_{0}$ of $\beta^{\prime}$ to $\gamma^{\prime}$. Suppose the minor gap of $L^{\prime}$ - if it exists - is not both periodic and nonsimplyconnected. Then any transverse intersections between $A_{0}$ and the minor leaf or sides of the minor gap of $L^{\prime}$ can be removed by good isotopy, and one of the following holds.

1. Each component of $A_{0}$ is contained in $L^{\prime}$ or a finite-sided gap of $L^{\prime}$.

2. There is a $\gamma$-invariant lamination $L$ containing $A_{0}$ and with all the other properties of the First Invariant-implies-Parameter Theorem, such that $L^{\prime} \subset L$. $W e$ can choose $L$ so that either of the following holds. The minor leaves of $L$, $L^{\prime}$ are the same, or at least one component of $A_{0}$ is contained in the forward orbit of the minor leaf or sides of the minor gap of $L$.

Proof: The proof is similar to that of the First Invariant-implies-Parameter Theorem, but we start by fixing a finite set $C_{0}$ of finite geodesics in the forward orbit of sides of the minor gap of $L^{\prime}$ - if this exists - or in the minor leaf of $L^{\prime}$, such that $\left(C_{0}, \gamma^{\prime}\right)$ has the minimal intersection property,

$$
C_{0} \subset\left(\sigma_{\gamma^{\prime}} \circ \bar{s}\right)^{-1} C_{0},
$$

and the minor leaf and sides of the minor gap (if the minor gap exists) are in the full orbit of $C_{0}$. Now we construct inductively $A_{n} \subset A_{n+1}, C_{n} \subset C_{n+1}$, with

$$
A_{n+1}=\left(\sigma_{\beta_{n}} \circ \bar{s}\right)^{-1} A_{n}, C_{n+1}=\left(\sigma_{\beta_{n}} \circ \bar{s}\right)^{-1} C_{n},
$$

where the definition of $\beta_{n}$ is slightly different from that in the First Theorem in some cases, and we do not define $C_{m}$ for $m \geq n$ if $C_{n}$ intersects $A_{n}$ transversally. For $n \geq 0, \beta_{n}$ is always defined so that either $\left(A_{n}, \beta_{n}\right)$ or $\left(A_{n} \cup C_{n}, \beta_{n}\right)$ has the minimal intersection property, depending on whether or not $C_{i}$ intersects $A_{i}$ transversally for some $i \leq n$. Similarly, $\beta_{n}$ and $\beta_{n+1}$ have the same intersections with $A_{n}$ or $A_{n} \cup C_{n}$. Also, $\beta_{n}(1)$ is always in the minor gap of $L^{\prime}$. The lamination $L$ is then obtained by taking the limit of $A_{n}$ or $A_{n} \cup C_{n}$, depending on whether or not $C_{n}$ intersects $A_{n}$ transversally. The Parameter Laminations Theorem $(1.16[\mathbf{R 2}])$ and $(7.1,7.3[\mathbf{R 2}])$ then show that, in fact, $C_{n}$ does not intersect $A_{n}$ transversally unless the minor gap of $L^{\prime}$ is periodic nonsimplyconnected. Every leaf of $L$ is contained in $L^{\prime}$ or a finite-sided gap of $L$ unless the minor gap of $L^{\prime}$ is periodic (and hence infinite-sided). 
There is a certain amount of choice in the construction. If some $A_{m}$ intersects $C_{m}$ transversally or the minor gap of $L^{\prime}$, then for some $N, \beta_{n}$ must always be close to the same component of $C_{N} \subset C_{n}$ or $A_{N} \subset A_{n}$ for $n \geq N$. Otherwise, $\beta_{n}$ must be close to the same component of $C_{N} \subset C_{n}$ for $n \geq N$ for some $N$. We can choose the component of $A_{N}$ to contain a component of $A_{0}$, if the minor gap of $L^{\prime}$ is periodic. We can satisfy these conditions, so we are done.

\subsection{Piecewise-geodesic Fixed Holed-spheres.}

Recall that $\bar{s}$ is a critically finite branched covering, and $X(\bar{s}) \backslash\{\infty\}=$ $X_{1}(\rho)=\left\{\rho^{i}(0): i \geq 0\right\}$ for any branched covering lamination map $\rho$ (for an invariant lamination containing $\left.\bar{L}_{r}\right)$.

Given an invariant lamination $L$ with lamination map $\rho$, let $A_{0}, B_{0}$ be the sets proved to exist in the Nonrational Lamination Map Theorem when $L$ is primitive and $\rho$ has a nondegenerate Levy cycle. It is clear, by considering the boundary of the set $A_{0} \cup B_{0}$, that the converse is true: if $\rho$ is a critically finite branched covering, and such that $A_{0}, B_{0}$ exist, then $\rho$ has a nondegenerate Levy cycle. The important thing to remember is that, if $m$ is the period of 0 under $\bar{s}$ (and $\rho$ ) then all segments of $A_{0}$ are eventually periodic of eventual oriented period $\leq m$, and if $A_{0} \cup B_{0}$ is not connected, the eventual oriented period is strictly less than the period of any periodic critical point. (I apologise for the change in use of $m$. We now revert to the letter conventions established in Chapter 1 of [R2]. The lettering in Chapters 1 to 4 of this paper is internally consistent as far as I can manage it, and that is the best I can do.) We shall call $A_{0} \cup B_{0}$ a piecewise-geodesic fixed holed-sphere, if it is connected, and, in general, a piecewise-geodesic periodic holed-sphere. Recall that $A_{n} \cup B_{n}$ is the component of $\rho^{-n}\left(A_{0} \cup B_{0}\right)$ containing $A_{0}$, and the properties satisfied by $A_{0} \cup B_{0}$ imply that $\overline{\cup A}$ is noncontractible in $\overline{\mathbf{C}} \backslash X_{1}(\rho)=\overline{\mathbf{C}} \backslash(X(\bar{s}) \backslash\{\infty\})$.

We also have the following, using the same notations as in the First Invariantimplies-Parameter Theorem and its proof.

Lemma. Let $\left(A_{0}, \beta\right)$ and $L$ be as in the First Invariant-implies-Parameter Theorem. Let $\rho$ be the lamination map of $L$. Also, let $B_{0}$ be a finite union of intervals in $S^{1} \backslash K$ with endpoints in $A_{0}$, such that the forward orbits of the components of $S^{1} \backslash K$ intersected by $\beta$ are not intersected by $B_{0}$. Then $\rho^{-n}\left(B_{0}\right) \subset S^{1}$ for all $n \geq 0$.

Similarly, let $\left(A_{0}, \beta\right), L^{\prime}$, with lamination map $\rho^{\prime}$, be as in the Second Invariant-implies-Parameter Theorem, with the same hypotheses as above. If the first conclusion of that theorem holds, $\rho^{-n}\left(B_{0}\right) \subset S^{1}$ for all $n$. If the second conclusion holds, $L$ can be chosen so that, in addition to the other properties, $\rho^{-n}\left(B_{0}\right) \subset S^{1}$ for all $n$. 
Proof: In both the First and Second Theorems, $\left(A_{n}, \beta_{n}\right)$ has the minimal intersection property. It suffices that, for all $n, \beta_{n} \cap B_{n}=\phi$, where $B_{n+1}=$ $\left(\sigma_{\beta_{n}} \circ \bar{s}\right)^{-1} B_{n}$. In the case of the First Theorem, this is obvious, by the condition on $\beta$, since $B_{n}=\left(\sigma_{\beta_{n}} \circ \bar{s}\right)^{-1} B_{n-1}=\left(\sigma_{\beta} \circ \bar{s}\right)^{-n} B_{0}=\bar{s}^{-n} B_{0}$. In the case of the Second Theorem, it is also clear, unless some component of $A_{n}$ is in the minor gap of $L^{\prime}$. In that case, we consider the forward orbits of those components of $S^{1} \backslash K$ which have to be crossed from the end of $\beta$ to reach that component. Then there is $N>n$ such that $\bar{s}^{-N} B_{0}$ intersects none of these components of $S^{1} \backslash K$. (Note that, by hypothesis, all components of $B_{0}$ lie in strictly preperiodic components of $S^{1} \backslash K$.) Take $\beta_{k}$ with endpoint close to the same side of $L^{\prime}$ for all $k<N$. Then, by the choice of $N$, we can choose $\beta_{N}$ having the same intersections with $A_{N-1}$ as $\beta_{N-1}$, so that $\beta_{N}$ does not intersect the forward orbit of $B_{N}$ and $\beta_{N}(1)$ is close to a component of $A_{N}$ in the minor gap. Then we take the end of $\beta_{k}$ close to this component of $A_{N}$ for all $k \geq N$.

\subsection{Moving to a Primitive Lamination.}

In Chapter 7 of [R2] we showed how to modify a lamination with eventually periodic minor gap (or leaf) to a primitive lamination. The corresponding lamination maps are then equivalent, and if one has a nondegenerate Levy cycle, they both do. Thus, by the Nonrational Lamination Map Theorem, if one has a piecewise-geodesic periodic holed-sphere, they both do. In fact, it is relatively easy to obtain the holed sphere for one directly from the other if one remembers the definition of primitive, but we omit the details.

\subsection{Admissible Points.}

The definition of admissible given in 1.17 of [R2] was slightly incorrect. There are several possible rather similar definitions. We give one here which avoids certain complications in the subsequent proof. The definition depends on a parameter lamination $\mathcal{L}=\mathcal{L}_{r}$ on $\widetilde{U}$, in which all leaves are either minor leaves or sides of minor gaps of invariant laminations containing all but countably many leaves of $\bar{L}_{r}$.

Types II, III and IV. Let $f$ be a degree two branched covering with critical points $c_{1}$ and $c_{2}$, with $c_{1}$ periodic. Recall from [R2] 1.2 that $f$ is type II if $c_{2}$ is in the same periodic orbit as $c_{1}$, type III if $c_{2}$ is in the full orbit of $c_{1}$ but not periodic, and type IV if $c_{2}$ is in a distinct periodic orbit. This includes all critically finite degree two branched coverings such that every critical point has a periodic critical point in its forward orbit.

A leaf $\widetilde{\mu}$ in $\mathcal{L}$ is admissible if it has a neighbourhood $V$ such that, whenever $L$ is a primitive invariant lamination with minor gap intersecting $V$, then $\rho_{L}$ does not have a nondegenerate Levy cycle. Note that if $\rho_{L}$ is type II, III or IV and has no nondegenerate Levy cycle, then $\rho_{L}$ is equivalent to a rational map. 
A point in Interior $(\widetilde{U})$ is admissible if it is either in an admissible leaf of $\mathcal{L}$ or in a gap with at least one admissible side. A point in $\partial \widetilde{U}$ is admissible if it has a neighbourhood whose intersection with Interior $(\widetilde{U})$ consists of admissible points. The set of admissible points is open by definition, and is denoted by $\widetilde{U}_{a d}$.

\section{9 .}

We are now ready to prove the Admissible Boundary Theorem, which we restate. See also the introduction of [R2]. Again, a very slight correction has been made. Recall that if $m$ is the period of $r$ under $x \mapsto 2 x \bmod 1$, then $m$ is also the period of 0 under $\bar{s}=\bar{s}_{r}$, and under lamination maps of all invariant laminations with minor leaves and minor gap sides in $\mathcal{L}_{r}$.

Admissible Boundary Theorem. Let $\widetilde{\nu}$ be a leaf in $\partial \widetilde{U}_{a d}$. Then either $\widetilde{\nu}$ is a side of the minor gap $G(L)$ of a primitive invariant lamination $L$, or there is a sequence $\left\{L_{n}\right\}$ of primitive invariant laminations with minor gaps $G\left(L_{n}\right)$ such that $\widetilde{\nu}=\lim _{n \rightarrow \infty} G\left(L_{n}\right)$. Also, the following hold. Let $\rho=\rho_{L}$ or $\rho_{L_{n}}$ be the lamination map of $L$ or $L_{n}$. Then $\rho$ is type $I V$, not equivalent to a rational map, and $\infty$ is periodic of period $\leq m$ under $\rho$. There is a piecewise-geodesic fixed holed sphere $A_{0} \cup B_{0}$ for $L$ (or $L_{n}$ ) such that $A_{0}$ intersects sides of the minor gap of $L$ (or $\left.L_{n}\right)$.

Proof: There are minor gaps of primitive invariant laminations intersecting any neighbourhood of $\widetilde{\nu}$ for which some of the corresponding lamination maps have nondegenerate Levy cycles, and some do not. So at least $L$ or $\left\{L_{n}\right\}$ exist with minor gaps $G(L), G\left(L_{n}\right)$ such that either $\widetilde{\nu}$ is a side of $G(L)$ or $\widetilde{\nu}=$ $\lim _{n \rightarrow \infty} G\left(L_{n}\right)$, and $\rho$ has a nondegenerate Levy cycle, where $\rho=\rho_{L}$ or $\rho_{L_{n}}$. In what follows, the point to remember is that two points on disjoint geodesics are close if the two geodesics have the same $S^{1}$-crossings for a considerable distance in both directions.

Write $\widetilde{\mu}$ for the minor leaf of $L_{n}$, or $\widetilde{\mu}=\widetilde{\nu}$ if $\widetilde{\nu}$ is a side of $G(L)$, so that $\widetilde{\nu}$ is approximated by leaves $\widetilde{\mu}$. Write $L$ rather than $L_{n}$ for the moment. The Nonrational Lamination Map Theorem implies that there is a piecewisegeodesic periodic holed-sphere $A_{0} \cup B_{0}$ for $L$. Let $A_{k} \cup B_{k}$ denote the subset of $\rho^{-k}\left(A_{0} \cup B_{0}\right)$ which is homotopic to $A_{0} \cup B_{0}$ in $\overline{\mathbf{C}} \backslash X(\rho)$ and such that each component of $A_{k}$ contains a component of $A_{0}$. (See 4.2.) The First Invariantimplies-Parameter Theorem can be interpreted as follows. Here, $\varepsilon, \delta$ depend only on a neighbourhood $V$ of a compact subset of $\widetilde{\nu}$ which $G(L)$ intersects. Write $\left.L\left(A_{k}, \beta\right)\right)$ for the lamination $L$ constructed from $\left(A_{k}, \beta\right)$ as in the First Theorem.

Given $\varepsilon>0$, there is $\delta>0$ such that, either $\widetilde{\mu} \cap V$ lies in an $\varepsilon$-neighbourhood of $G\left(L\left(A_{k}, \beta\right)\right)$, or no $A_{k}$ passes within $\delta$ of $V \cap \widetilde{\mu}$. 
In fact, given $\varepsilon>0$, the first possibility must hold for some $\widetilde{\mu}$. Otherwise, the Second Invariant-implies-Parameter Theorem gives the following.

There is $\eta>0$, which depends on $\delta$, and hence on $\varepsilon$, such that every primitive lamination $L^{\prime}$ with minor gap passing within $\eta$ of $\widetilde{\mu} \cap V$ satisfies one of the following.

a) $A_{k}$ (for any $k$ ) is contained in leaves or finite sided gaps of $L^{\prime}$.

b) $L^{\prime} \subset L^{\prime \prime}$ with $A_{k} \subset L^{\prime \prime}$ such that $L^{\prime \prime}$ and $L^{\prime}$ have the same minor leaf.

Xc) $L^{\prime}$ has a nonsimply-connected minor gap.

By Lemma 5.6 and 5.7, we can choose $L^{\prime \prime}$ so that $A_{k} \cup B_{k}$ is a piecewisegeodesic periodic holed sphere for $L^{\prime \prime}$, and then modify to a primitive lamination. (The hypothesis of 5.6 is satisfied, because $A_{0} \cup B_{0}$ is a piecewise-geodesic periodic holed-sphere for $L$.) If $\widetilde{\mu}$ is sufficiently close to $\widetilde{\nu}$, this implies $\widetilde{\nu}$ is in the interior of $\widetilde{U} \backslash \widetilde{U}_{a d}$, giving a contradiction.

Now using laminations $L\left(A_{k}, \beta\right)$ instead of the original $L_{n}$ (or $L$ ), the corresponding lamination maps are all type IV, not equivalent to rational maps. If the other property (concerning the piecewise-geodesic fixed holed-sphere) is not satisfied, then the period of $\infty$, for all the maps in the sequence, is less then $m$, by the Nonrational Lamination Map Theorem. We can then repeat the entire process. After finitely many repeats, we obtain a sequence with the required properties.

\subsection{Preliminaries to the Admissibility Proposition.}

Every leaf intersecting the admissible set is contained in it. If a lamination map $\rho_{L}$ of a lamination $L$ is critically finite and equivalent to a rational map, one would expect all sides of the minor gap to be in the admissible set. Actually, if $\rho_{L}$ is equivalent to a polynomial this cannot quite be true - because then some tunings of $\rho_{L}$ (with minor gaps contained in the minor gap of $L$ ) will not be equivalent to rational maps. But if $\rho_{L}$ is equivalent to a polynomial and hence to $\bar{s}_{r}$ for some $r$, then $\rho_{L}$ is associated to the gap of $\mathcal{L}_{r}$ containing 0 , which is admissible. Perhaps the easiest degree two rational maps to study are matings and captures, originally defined by Douady and Hubbard [D] and Wittner [W]. They are also described in 1.19 of [R2], where a subset $\widetilde{U}_{m c}$ of $\widetilde{U}$ is described in which all matings and captures are represented, and a description is given (without proof) of $\widetilde{U}_{a d} \cap \widetilde{U}_{m c}$. This set is connected and contains up to equivalence all matings and captures which are equivalent to rational maps. For matings, the description is the result of Tan Lei's thesis [TL]. In both cases, it follows from the more general Admissible Boundary Theorem, or even the Nonrational Lamination Map Theorem. A simplifying factor is that, if $\widetilde{\mu}$ and $\widetilde{\mu}^{\prime}$ are two minor leaves in $\widetilde{U}_{m c}$ of laminations $L$ and $L^{\prime}$, and $\widetilde{\mu}$ separates $\widetilde{\mu}^{\prime}$ from 0 , then all periodic leaves in $L$ are in $L^{\prime}$. So now we state the Admissibility Proposition, in a slightly different, but equivalent form to that given in 1.17 of 
[R2], given that the definition of admissibility has been altered in 5.8 from that of 1.17 of [R2].

The Admissibility Proposition. Let $f$ be a critically finite degree two rational map which is not equivalent to a polynomial, mating or capture. Then whenever $L$ is an invariant lamination with minor leaf or gap in $\widetilde{U}$ and the lamination map $\rho_{L}$ is equivalent to $f$, the minor leaf or gap lies in the admissibility set $\widetilde{U}_{a d}$.

\subsection{The Inadmissible Type II Case.}

\section{Lemma.}

Let $L$ be an invariant lamination with type II lamination map $\rho_{L}$ which is equivalent to a rational map $f$. Let $L^{\prime}$ be the lamination $L \cup \bar{L}_{r}$ with type IV lamination map $\rho^{\prime}$ which is not equivalent to a rational map. Then $\rho^{\prime}$ is equivalent to a mating and $\rho_{L}$ is equivalent to a capture.

Proof: Let $G_{\infty}$ be the gap of $L$ containing $\infty$, and let $\widetilde{G}$ be a lift to the universal cover of $\overline{\mathbf{C}} \backslash K$, with projection $\pi: \overline{\widetilde{G}} \rightarrow \bar{G}_{\infty}$. Let $\widetilde{\rho}$ be a lift of $\rho_{L}^{m} \mid G_{\infty}$ fixing $\widetilde{G}$, and extending to $\overline{\widetilde{G}}$, if $m$ is the period of $G_{\infty}$ under $\rho_{L}$. Let $\Phi: \overline{\mathbf{C}} \rightarrow \overline{\mathbf{C}}$ be the semiconjugacy satisfying $\Phi \circ \rho_{L}=f \circ \Phi$. Let $\varphi_{1}:\{z:|z| \leq 1\} \rightarrow \overline{U_{2}(f)}$ and $\varphi_{2}: \overline{\widetilde{G}} \rightarrow\{z:|z| \leq 1\}$ satisfy

$$
\begin{gathered}
\varphi_{1} \circ \varphi_{2}=\Phi \circ \pi, \\
\varphi_{1}\left(z^{4}\right)=f \circ \varphi_{1}(z), \\
\varphi_{2} \circ \widetilde{\rho}(z)=\left(\varphi_{2}(z)\right)^{4}
\end{gathered}
$$

for all $z$. Let $\omega_{1}, \omega_{2}$ be the cube roots of unity which are the images under $\varphi_{2}$ of the endpoints of the lift to $\widetilde{G}$ of $\ell$, where $\ell$ is the unique leaf of $\bar{L}_{r} \backslash L$ of oriented period $m$ in $G_{\infty}$. Let $\alpha$ be an arc in $\overline{U_{2}(f)}$ which is the image under $\varphi_{1}$ of an arc in $\{z:|z| \leq 1\}$ joining $\omega_{1}$ and $\omega_{2}$. We can choose $\alpha$, and can perturb $f$ to a critically finite branched covering $f^{\prime}$, so that $f^{\prime}=f$ outside a neighbourhood of the critical orbit of $f, f^{\prime}$ preserves the set $\cup_{i=0}^{m-1} f^{\prime i} \alpha, f^{\prime i} \alpha \subset f^{\prime i} U_{2}(f)=f^{i} U_{2}(f)$ separates the two points of $X\left(f^{\prime}\right)$ in $f^{i} U_{2}(f)$, and $f^{\prime} \simeq \rho^{\prime}$. So $f^{\prime}$ admits a minimal Levy cycle $\Gamma$, that is, one with only one complementary component which is not a disc. (See 1.6 of [R2].) Let $P$ be the closure of the nondisc component of $\overline{\mathbf{C}} \backslash(\cup \Gamma)$. Let $F_{0}: P \times[0,1] \rightarrow \overline{\mathbf{C}} \backslash X\left(f^{\prime}\right)$ be a homotopy between $P$ and a component of $f^{\prime-1}(P)$ with $f^{\prime} \circ F_{0}(x, 1)=F_{0}(x, 0)$. (This exists for a minimal Levy cycle.) We can assume $F_{0}(P \times[0,1]) \cap\left(\cup_{i \geq 0} f^{i}\left(U_{2}(f)\right) \subset \cup_{i \geq 0} f^{\prime i} \alpha\right.$. Defining $F_{n}$ inductively by $f^{\prime} \circ F_{n+1}=F_{n}, F_{n+1}(x, 0)=F_{n}(x, 1)$, and taking limits, we can assume $\cup \Gamma \subset \cup_{i \geq 0} \overline{f^{\prime i} \alpha}$, if we drop the assumption that the loops of 
$\Gamma$ are embedded and disjoint. (See Section 8 of [R1] for a similar construction.) This set must contain at least one point fixed by $f^{\prime}$ by the Lefschetz Theorem. So one endpoint of $\alpha$ must be fixed by $f$ and by $f^{\prime}$. The endpoints of $\alpha$ must be distinct if $m \geq 3$, for topological reasons, but also if $m=2$, since then $f$ is $z \mapsto z^{-2}$, up to Möbius conjugation. Then the second endpoint must also be fixed. Then we can find a circle $\Gamma^{\prime}$ invariant up to homotopy under $f^{\prime}$, and separating the critical orbits, which is equivalent to $f^{\prime}$ being a mating. See Diagram 5.

\section{Diagram 5}

Then

$$
f \simeq \sigma_{\bar{\eta}} \circ \sigma_{\gamma} \circ f^{\prime}
$$

where $\gamma, \eta$ are loops crossing $\Gamma^{\prime}$ once each, (where $\bar{\eta}$, as usual, is $\eta$ reversed), and $f^{\prime}(\eta)$ is homotopic to $\gamma$ in $\overline{\mathbf{C}} \backslash X\left(f^{\prime}\right)$. Let $f_{0}$ be the polynomial obtained by modifying $f^{\prime}$ on the component of $\overline{\mathbf{C}} \backslash \Gamma^{\prime}$ containing $c_{2}\left(f^{\prime}\right)$, so that $c_{2}\left(f_{0}\right)$ is

fixed. Up to homotopy in $\overline{\mathbf{C}} \backslash X\left(f_{0}\right), f_{0}^{-1}$ preserves $\Gamma^{\prime}$ and the components of $D \backslash\left(\cup\left(f^{\prime}\right)^{i} \alpha\right)$, where $D$ is the component of $\overline{\mathbf{C}} \backslash \Gamma$ containing $c_{1}$. Hence, $f_{0}$ is a polynomial (corresponding to a minimal minor leaf in $Q M L$ : see 1.10 of [R2]). Then, modifying $\gamma$ and $\eta$ also, there are paths $\beta$ and $\zeta$ in $\overline{\mathbf{C}} \backslash X\left(f_{0}\right)$ with $f_{0}(\zeta)$ homotopic in $\overline{\mathbf{C}} \backslash X\left(f_{0}\right)$ and

$$
f \simeq \sigma_{\bar{\zeta}} \circ \sigma_{\beta} \circ f_{0}
$$

as required. 


\subsection{Definition of $\mathrm{W}_{1}, \mathrm{~W}_{2}$.}

Let $f$ be a critically finite rational map with numbered critical points $c_{1}, c_{2}$. Let $c_{1}$ be periodic, and suppose $f$ is not type II. Then we define sets $W_{1}, W_{2}$ as follows. If $f$ is not type III or IV (so that, in particular, $c_{2}$ is not periodic) then $W_{1}=W_{2}$ is simply $\left\{f^{i}\left(c_{2}\right): i>0\right\}$. If $f$ is type III, then $W_{1}=W_{1}(x)=W_{2}$ is $\left\{f^{i}(x): i \geq 0\right\}$ for an eventually periodic point $x \in \partial f\left(U_{2}\right)$. If $f$ is type IV, then $W_{2}$ is the closure of the (finite) union of components of $\overline{\mathbf{C}} \backslash J(f)$ which intersect the forward orbit of $c_{2}$, and $W_{1}$ is the smallest set with the following properties.

1. $W_{2} \subset W_{1}$

2. $W_{1}$ contains $\bar{U}$, whenever $U$ is a component of $\overline{\mathbf{C}} \backslash J(f)$ in the full orbit of $U_{2}(f)$ with $\bar{U} \cap W_{1} \neq \phi$.

Then $W_{1}$ has finitely many components. By 5.4 of [R2], if $U$ and $V$ are in the same period $p$ orbit of components of $\overline{\mathbf{C}} \backslash J(f)$, with $U \neq V$, then $\bar{U} \cap \bar{V}$ consists of at most one point, which is fixed by $f^{p}$.

\subsection{Lemma.}

Let $f$ be type $I V$. All components of $\bar{W}_{1}$ are simply connected, unless $f$ is equivalent to a polynomial or mating

Proof: Suppose that components of $\bar{W}_{1}$ are not simply connected and that $f$ is not a polynomial, so that $c_{2}(f)$ has period $p>1$. By 5.4 of [R2], $\bar{U}$ is simply connected for any component $U$ of $\overline{\mathbf{C}} \backslash J(f)$. So the components of $W_{1}$ are larger than the closures of components of $\overline{\mathbf{C}} \backslash J(f)$. There is $x \in \cap_{j \geq 0} f^{j t} \overline{U_{2}(f)}$ with $x$ of period $t<p$. Let $\gamma$ be an embedded circle enclosing $x$ and lying in the union of the $f^{j t} U_{2}(f)$ and a neighbourhood of $x$, and enclosing $x$ and $f^{j t}\left(c_{2}\right)(j \geq 0)$. Then $\gamma$ is isotopic to a component $\gamma_{1}$ of $f^{-t}(\gamma)$ in $\overline{\mathbf{C}} \backslash X(f)$, with $f^{t} \mid \gamma_{1}$ degree 2. Then $f$ must be a tuning $g \vdash_{c_{2}} h$ of a type IV rational map $g$ (see [R2] 1.20), where $h$ is a polynomial for which the boundaries of the attractive basins have a common fixed point. It is possible to define a branched covering $f_{1}$ which is equivalent to $f$ and equal to $g$ on $J(g)$. Moreover, the Semiconjugacy Proposition of 4.1 of [R2] implies that there is $\varphi: \overline{\mathbf{C}} \rightarrow \overline{\mathbf{C}}$ with $\varphi \circ f_{1}=f \circ \varphi$, and $\varphi$ is a limit of homeomorphisms. Since $f_{1} \mid J(g)$ is expanding, each set $\varphi^{-1} x$, which is connected and simply- connected, has finite intersection with $J(g)$. (See 4.6 of [R2].) Then $\bar{W}_{1}=\varphi\left(\cup_{i \geq 0} g^{i} \overline{U_{2}(g)}\right)$. So components of $\cup_{i \geq 0} g^{i} \overline{U_{2}(g)}$ are not simply connected. Then by 5.4 of [R2], $g$ is a polynomial. Then $f$ is a mating, since the tuning of a polynomial is a mating.

\subsection{Lemma.}

There is an arbitrarily small neighbourhood $W$ of $W_{1}$ and such that, for all $n$, each component of $f^{-n} W$ is either contained in, or disjoint from, $W$. 
Proof: We start with a neighbourhood $W^{\prime}$ of $W_{1}$ such that $W^{\prime}$ contains the components of $f^{-p} W^{\prime}$ intersecting $W_{1}$ (if $p$ is the least integer $>0$ with $\left.f^{p} W_{1}=W_{1}\right)$. The idea is then to reduce $W^{\prime}$ to obtain $W$, without violating the neighbourhood condition. We see that there is $\delta>0$ with the following property.

a) If $W^{\prime \prime}$ is a component of $f^{-n} W^{\prime}$ for some $n, W^{\prime \prime}$ does not contain $W_{1}$ and has diameter $\varepsilon$, then $\operatorname{dist}\left(W_{1}, W^{\prime \prime}\right)>\delta \varepsilon$

Of course, this property remains true if we make $W^{\prime}$ even smaller. Given any $\eta>0$, we can choose $W^{\prime}$ so close to $W_{1}$ that the following is true.

b) If $W^{\prime \prime}$ is as in a), and $W^{\prime \prime} \cap W^{\prime} \neq \phi$, then $\varepsilon<\eta$.

There is $M>0$ such that the following is true (and remains true if $W^{\prime}$ is made smaller).

c) For all $z, w$ in the domain of a local inverse $S$ of $f^{n}$ defined on a subset of $W^{\prime} \backslash W_{1}$,

$$
\frac{1}{M} \leq \frac{\left|S^{\prime}(z)\right|}{\left|S^{\prime}(w)\right|} \leq M
$$

It follows that if $W^{\prime}$ is close enough to $W_{1}$, then given $\nu>0$,

d) if $W^{\prime}, W^{\prime \prime}$ are as in a) and $W^{\prime} \cap W^{\prime \prime} \neq \phi$ and $W_{n}^{\prime}, W_{n}^{\prime \prime}$ are preimages under $f^{n}$ with $W_{n}^{\prime} \cap W_{n}^{\prime \prime} \neq \phi$, then $W_{n}^{\prime \prime}$ is contained in the diameter $\left(W_{n}^{\prime}\right) \times \nu$ neighbourhood of $W_{n}^{\prime}$ and has diameter $<\operatorname{diameter}\left(W_{n}^{\prime}\right) \times \nu$.

Now we inductively define $T_{0}=W^{\prime}$, and $T_{n+1}$ is $T_{n} \backslash R_{n}$, where $R_{n}$ is the union of those components of $f^{-k} T_{n}$ (for all $k$ ) which intersect, but are not contained in, $T_{n}$. We take $W=\cap_{n} T_{n}$. Then $W$ has the required properties provided $W^{\prime}$ is close enough to $W_{1}$ - because every component of $R_{n+1}$ intersects a component of $R_{n}$. Hence we see inductively that every component of $R_{n}$ is distance $\leq$

$$
\sum_{i=1}^{n} \nu^{i} \times \operatorname{diameter}(C)
$$

from a component $C$ of $R_{0}$ which is itself distance $>\delta \times$ diameter $(C)$ from $W_{2}$, by a). So we only need to take $\frac{\nu}{1-\nu}<\delta$. By its definition $T_{n}$ contains the component of $f^{-p}\left(T_{n}\right)$ containing $W_{1}$, so $W$ contains the component of $f^{-p} W$ containing $W_{1}$.

\subsection{Proof of the Admissibility Proposition.}

Let $f$ be a critically finite rational map which is not equivalent to a polynomial, mating or capture. Let $L$ be an invariant lamination with minor leaf or gap in $\widetilde{U}$, such that the lamination map $\rho$ is equivalent to $f$. By 5.11 , if $f$ is type II, the lamination map for the lamination $L \cup \bar{L}_{r}$ is equivalent to a rational map of type IV. So now we can assume that $f$ is not type II. Then the 
Semiconjugacy Proposition 4.1 of [R2] gives a semiconjugacy $\Phi: \overline{\mathbf{C}} \rightarrow \overline{\mathbf{C}}$ such that $\Phi \circ \rho=f \circ \Phi, \Phi$ is a limit of homeomorphisms and collapses each leaf and finite sided gap of $L$ to a point. If $f$ is type III, let $x$ be the image under $\Phi$ of the minor leaf of $L$. Then let $W_{1}$ be as in 5.12, with $W_{1}=W_{1}(x)$ if $f$ is type III. By $5.13, \bar{W}_{1}$ is simply-connected. Let $W$ be as in 5.14 , and we can choose $W$ close enough to $W_{1}$ so that $\bar{W}$ is contractible in $\overline{\mathbf{C}} \backslash\left\{f^{i}\left(c_{1}\right): i \geq 0\right\}$. Put

$$
V=\pi^{-1}\left(\left(\overline{\mathbf{C}} \backslash\left(K \cup\left(\cup \bar{L}_{r}\right)\right)\right) \cap \Phi^{-1} W\right) \subset \widetilde{U},
$$

where $\pi$ is the universal covering map. Then $\pi(\bar{V})$ is contractible in $\overline{\mathbf{C}} \backslash\left\{\bar{s}^{i}(0)\right.$ : $i \geq 0\}$ and is a neighbourhood of the minor leaf of $L$, and of the minor gap if this exists, such that $\pi(V)$ contains any component of $\rho^{-n}(V)$ it intersects, for any $n \geq 0$. We claim that the proof of the Admissibility Proposition is completed by the following lemma, using the notation established here.

Lemma. Let an integer $m>0$ be given. Let $A$ denote the set of leaves of $L$ of period $\leq m$. Let $V^{\prime} \subset \vee$ be a neighbourhood of the minor leaf or minor gap. Let $L^{\prime}$ be any primitive invariant lamination with minor gap intersecting $V^{\prime}$. Let $A^{\prime}$ be the set of leaves of $L^{\prime}$ of period $\leq m$. Then if $V^{\prime}$ is sufficiently small, every leaf of $A^{\prime}$ is in $A$, or a finite-sided gap of $L$ bounded by leaves of $A$, or in $\pi(V)$.

We see that the lemma implies the proposition as follows. Let $m$ be the period of $r$ under $x \mapsto 2 x \bmod 1$, so that $m$ is the period of 0 under $\bar{s}_{r}=\bar{s}, \rho$, $\rho^{\prime}$, and, indeed, under $\tau$, where $\tau$ is any lamination map of an invariant lamination with minor leaf in $\mathcal{L}_{r}$. Suppose the lamination map $\rho^{\prime}$ of $L^{\prime}$ is a critically finite branched covering. By the Nonrational Lamination Map Theorem, $\rho^{\prime}$ does not have a nondegenerate Levy cycle provided $\overline{A^{\prime}}$ is contractible in $\overline{\mathbf{C}} \backslash X(\bar{s})$ (to finitely many points). But this is true, because $\overline{A \cup \pi(V)}$ is contractible in $\overline{\mathbf{C}} \backslash X(\bar{s})$, since $A$ maps to points under $\Phi$ and $\overline{\pi(V)}$ maps to $\bar{W}$.

\subsection{Proof of Lemma 5.15.}

Let $C$ be an orbit in $A^{\prime}$. We have $C=\cup_{n \geq 0} C_{n}$, where $C_{n} \subset C_{n+1} \subset$ $\rho^{\prime-1} C_{n}$ and the number of segments in $C_{0}$ can be bounded independently of $L^{\prime}$. (The bound depends on $V^{\prime}$.) By taking $V^{\prime}$ sufficiently small, given $t$, we can assume that any geodesic of $L$ of $\geq t$ segments intersecting $\pi\left(V^{\prime}\right)$ cannot be isotoped into $\partial \pi(V)$ by good isotopy. We can also take $V^{\prime}$ small enough that any such $C_{0}$ does not lift to lie strictly between the minor leaves of gaps of $L, L^{\prime}$, up to good isotopy. Take any $k$ such that $C_{i}$ does not intersect $\pi\left(V^{\prime}\right)$ for $i<k$. By the Second Invariant-implies-Parameter Theorem (with $L$ replacing $L^{\prime}$ ), $C_{k}$ does not intersect $L$ transversally. So either $C_{k} \subset \pi(V)$, or $C_{k} \cap \pi(V)=\phi$, and then $C_{k}$ lies in leaves or finite-sided gaps of $L$ up to good isotopy. But $\rho^{-1}=\rho^{-1}$ outside $\pi(V)$, and $\rho^{-k}(\pi(V))=\rho^{-k}(\pi(V))$ for all $k \geq 0$. So either 
$C \subset \cup_{n \geq 0} \rho^{\prime-n} C_{0}$ and $C \subset \pi(V)$ or, for all $k, C_{k}$ lies in leaves or finite-sided gaps of $L$ up to good isotopy, and hence so does $C$. 


\section{References}

[D] Douady, A.: Systèmes dynamiques holomorphes. Séminaire Bourbaki 1983, Astérisque 105/6 (1983), 39-63.

[D-H1] Douady, A. and Hubbard, J.H.: Etudes dynamiques des polynômes complexes, avec la collaboration de P. Lavaurs, Tan Lei, P. Sentenac. Parts I and II, Publications Mathématiques d'Orsay, 1985.

[D-H2] Douady, A. and Hubbard, J.H.: Itérations des polynômes quadratiques complexes. C.R.Acad.Sci. Paris, Série I, t.294 (1982), 123-126.

[D-H3] Douady, A. and Hubbard, J.H.: A proof of Thurston's topological characterization of rational functions. Mittag-Leffler preprint, 1985.

[F-L-P] Fahti, A., Laudenbach, F., and Poénaru, V.: Travaux de Thurston sur les Surfaces. Astérisque 66-67 (1989).

[Fr] Franks, J.: Anosov Diffeomorphisms. Proc. Symposia in Pure Math., 14 (1968), 61-93.

[R1] Rees, M.: Components of degree two hyperbolic rational maps. Invent. Math., 100 (1990), 357-382.

[R2] Rees, M.: A Partial Description of the Parameter Space of Rational Maps of Degree Two. Part 1. To appear in Acta Math.

[S] Shishikura, M.: In preparation.

[T] Thurston, W.P.: On the Combinatorics of Iterated Rational Maps. Preprint, Princteton University and I.A.S., 1985.

[TL] Tan Lei: Accouplements des polynômes complexes. Thèse, Université de Paris-Sud, Orsay, 1987.

[W] Wittner, B.: On the bifurcation loci of rational maps of degree two. Thesis, Cornell University, 1988. 